\title{
261.
}

\section{ON THE CONIC OF FIVE-POINTIC CONTACT AT ANY POINT OF A PLANE CURVE.}

[From the Philosophical Transactions of the Royal Society of London, vol. cxuIx. (for the year 1859), pp. 371-400. Received March 1,-Read March 24, 1859.]

THE tangent is a line passing through two consecutive points of a plane curve, and we may in like manner consider the conic which passes through five consecutive points of a plane curve; and as there are certain singular points, viz. the points of inflexion where three consecutive points of the curve lie in a line, so there are singular points where six consecutive points of the curve lie in a conic. In the particular case where the given curve is a cubic, the last-mentioned species of singular points have been considered by Plücker and Steiner, and in the same particular case, the theory of the conic of five-pointic contact has recently been established by $\mathrm{Mr}$ Salmon. But the general case, where the curve is of any order whatever, has not so far as I am aware been hitherto considered;-the establishment of this theory is the object of the present memoir.

\section{Investigation of the Equation of the Conic of Five-pointic Contact.}

1. I take $(X, Y, Z)$ as current coordinates, and I represent the equation of the given curve by

$$
\Upsilon=(* \gamma X, Y, Z)^{m}=0 .
$$

Let $(x, y, z)$ be the coordinates of a given point on the curve, and let $U=(* \gamma x, y, z)^{n}$ be what $\Upsilon$ becomes when $(x, y, z)$ are written in the place of $(X, Y, Z)$; we have therefore $U=0$ as a condition satisfied by the coordinates of the point in question.

2. Write for shortness

$$
\begin{aligned}
& D U=\left(X \partial_{x}+Y \partial_{y}+Z \partial_{z}\right) U, \\
& D^{2} U=\left(X \partial_{x}+Y \partial_{y}+Z \partial_{z}\right)^{2} U,
\end{aligned}
$$

and let $\Pi=a X+b Y+c Z=0$ be the equation of a line. It is easy to see that

$$
D^{2} U-\Pi . D U=0
$$


will be the equation of a conic having an ordinary (two-pointic) contact with the curve at the point $(x, y, z)$. In fact the equation $D U=0$ is that of the tangent at the point in question, and the equation $D^{2} U=0$ is that of the penultimate polar (or polar conic) of the point, which conic is touched by the tangent; the assumed equation represents therefore a conic having an ordinary (two-pointic) contact with the polar conic, and therefore with the curve. It may be added that the two conics intersect besides in a pair of points, and that the line joining these, or common chord of the two conics, is the line represented by the equation $\Pi=0$; and this being so, the constants $(a, b, c)$ of the line $\Pi=0$ can be so determined as to give rise to a five-pointic contact.

3. Consider the coordinates of a point of the curve as functions of a single variable parameter; then for the present purpose the coordinates of a point consecutive to $(x, y, z)$ may be taken to be

$$
\begin{aligned}
& x+d x+\frac{1}{2} d^{2} x+\frac{1}{6} d^{3} x+\frac{1}{24} d^{4} x, \\
& y+d y+\frac{1}{2} d^{2} y+\frac{1}{6} d^{3} y+\frac{1}{24} d^{4} y, \\
& z+d z+\frac{1}{2} d^{2} z+\frac{1}{6} d^{3} z+\frac{1}{24} d^{4} z,
\end{aligned}
$$

values which, substituted for $X, Y, Z$, must satisfy the equations

$$
\Upsilon=0, \quad D^{2} U-\Pi . D U=0 .
$$

4. I write for shortness

$$
\begin{aligned}
& \partial_{1}=d x \partial_{x}+d y \partial_{y}+d z \partial_{z}, \\
& \partial_{2}=d^{2} x \partial_{x}+d^{2} y \partial_{y}+d^{2} z \partial_{z}, \\
& \partial_{3}=d^{3} x \partial_{x}+d^{3} y \partial_{y}+d^{3} z \partial_{z}, \\
& \partial_{4}=d^{4} x \partial_{x}+d^{4} y \partial_{y}+d^{4} z \partial_{z},
\end{aligned}
$$

then the consecutive value of $\Upsilon$ is

$$
\text { exp. }\left(\partial_{1}+\frac{1}{2} \partial_{2}+\frac{1}{6} \partial_{3}+\frac{1}{24} \partial_{4}\right) U
$$

( $\operatorname{Read}$ exp. $z$, exponential of $\left.z,=e^{z}\right)$, which is

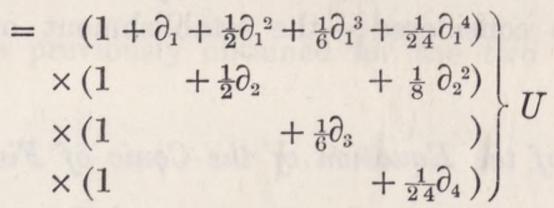

$$
\begin{aligned}
& =1+\partial_{1}+\frac{1}{2} \partial_{1}{ }^{2}+\frac{1}{6} \partial_{1}{ }^{3}+\frac{1}{24} \partial_{1}{ }^{4} \\
& +\frac{1}{2} \partial_{2}+\frac{1}{2} \partial_{1} \partial_{2}+\frac{1}{4} \partial_{1}^{2} \partial_{2} \\
& +\frac{1}{8} \partial_{2}{ }^{2} \\
& +\frac{1}{6} \partial_{3}+\frac{1}{6} \partial_{1} \partial_{3} \\
& \begin{array}{r}
\quad U \\
+\quad \partial_{1} U
\end{array} \\
& +\frac{1}{24} \partial_{4} \\
& +\frac{1}{2}\left(\partial_{1}{ }^{2}+\partial_{2}\right) U \\
& +\frac{1}{6}\left(\partial_{1}{ }^{3}+3 \partial_{1} \partial_{2}+\partial_{3}\right) U \\
& +\frac{1}{24}\left(\partial_{1}{ }^{4}+6 \partial_{1}{ }^{2} \partial_{2}+4 \partial_{1} \partial_{3}+3 \partial_{2}{ }^{2}+\partial_{4}\right) U \text {, }
\end{aligned}
$$


the several terms of which must respectively vanish, and we have therefore

$$
\begin{aligned}
U & =0, \\
\partial_{1} U & =0 \\
\partial_{2} U & =-\partial_{1}{ }^{2} U \\
\partial_{3} U & =-\left(\partial_{1}{ }^{3}+3 \partial_{1} \partial_{2}\right) U, \\
\partial_{4} U & =-\left(\partial_{1}{ }^{4}+6 \partial_{1}{ }^{2} \partial_{2}+4 \partial_{1} \partial_{3}+3 \partial_{2}{ }^{2}\right) U .
\end{aligned}
$$

5. Next, preparing to substitute in the equation

$$
D^{2} U-\Pi . D U=0 \text {, }
$$

the consecutive value of $D U$ is

where

$$
\begin{aligned}
& \left(x+d x+\frac{1}{2} d^{2} x+\frac{1}{6} d^{3} x+\frac{1}{24} d^{4} x\right) \partial_{x} U+\& c . \\
= & \left(\partial_{0}+\partial_{1}+\frac{1}{2} \partial_{2}+\frac{1}{6} \partial_{3}+\frac{1}{24} \partial_{4}\right) \quad U,
\end{aligned}
$$

$$
\partial_{0} U=\left(x \partial_{x}+y \partial_{y}+z \partial_{z}\right) U=m U \text {. }
$$

Reducing by the above results, the consecutive value of $D U$ is

$$
=-\frac{1}{2} \partial_{1}{ }^{2} U-\frac{1}{6}\left(\partial_{1}{ }^{3}+3 \partial_{1} \partial_{2}\right) U-\frac{1}{24}\left(\partial_{1}{ }^{4}+6 \partial_{1}{ }^{2} \partial_{2}+4 \partial_{1} \partial_{3}+3 \partial_{2}{ }^{2}\right) U .
$$

6. Hence also writing

$$
\begin{aligned}
P & =a x+b y+c z, \\
\partial_{1} P & =a d x+b d y+c d z, \\
\partial_{2} P & =a d^{2} x+b d^{2} y+c d^{2} z,
\end{aligned}
$$

the consecutive value of $-\Pi D U$ is $-\left(P+\partial_{1} P+\frac{1}{2} \partial_{2} P\right)$ multiplied into the consecutive value of $D U$, and the product is

$$
\begin{aligned}
= & P \cdot \frac{1}{2} \partial_{1}{ }^{2} U \\
& +P \cdot \frac{1}{6}\left(\partial_{1}{ }^{3}+3 \partial_{1} \partial_{2}\right) U \quad+\partial_{1} P \cdot \frac{1}{2} \partial_{1}{ }^{2} U \\
& +P \cdot \frac{1}{24}\left(\partial_{1}{ }^{4}+6 \partial_{1}{ }^{2} \partial_{2}+4 \partial_{1} \partial_{3}+3 \partial_{2}{ }^{2}\right) U+\partial_{1} P \cdot \frac{1}{6}\left(\partial_{1}{ }^{3}+3 \partial_{1} \partial_{2}\right) U+\frac{1}{2} \partial_{2} P \cdot \frac{1}{2} \partial_{1}{ }^{2} U .
\end{aligned}
$$

7. The consecutive value of $D^{2} U$ is

$$
\left.\begin{array}{rl}
= & \left(x+d x+\frac{1}{2} d^{2} x+\frac{1}{6} d^{3} x+\frac{1}{24} d^{4} x\right)^{2} \partial_{x}^{2} U+\& c . \\
= & x^{2} \\
& +2 x d x \\
& +x^{2} d x+(d x)^{2} \\
& +\frac{1}{3} x d^{3} x+d x d^{2} x \\
& +\frac{1}{12} x d^{4} x+\frac{1}{3} d x d^{3} x+\frac{1}{4}\left(d^{2} x\right)^{2}
\end{array}\right\}
$$

C. IV. 
which is

$$
\begin{aligned}
& =\partial_{0}{ }^{2} \\
& +2 \partial_{0} \partial_{1} \\
& +\partial_{0} \partial_{2}+\partial_{1}^{2} \\
& +\frac{1}{3} \partial_{0} \partial_{3}+\partial_{1} \partial_{2} \\
& +\frac{1}{12} \partial_{0} \partial_{4}+\frac{1}{3} \partial_{1} \partial_{3}+\frac{1}{4} \partial_{2}{ }^{2}
\end{aligned}
$$

and observing that

$$
\begin{aligned}
& \partial_{0}{ }^{2} U=m(m-1) U, \\
& \partial_{0} \partial_{1} U=(m-1) \partial_{1} U, \\
& \partial_{0} \partial_{2} U=(m-1) \partial_{2} U, \\
& \partial_{0} \partial_{3} U=(m-1) \partial_{3} U, \\
& \partial_{0} \partial_{4} U=(m-1) \partial_{4} U,
\end{aligned}
$$

and reducing as before, the consecutive value of $D^{2} U$ is

$$
\begin{aligned}
= & -(m-2) \partial_{1}{ }^{2} U \\
& -\frac{1}{3}\left[(m-1) \partial_{1}{ }^{3}+3(m-2) \partial_{1} \partial_{2}\right] U \\
& -\frac{1}{12}\left[(m-1)\left(\partial_{1}{ }^{4}+6 \partial_{1}{ }^{2} \partial_{2}\right)+(m-2)\left(4 \partial_{1} \partial_{3}+3 \partial_{2}{ }^{2}\right)\right] U .
\end{aligned}
$$

8. Substituting in the equation $D^{2} U-\Pi \cdot D U=0$, we obtain as the conditions of a five-pointic contact

$$
-(m-2) \partial_{1}^{2} U+P \cdot \frac{1}{2} \partial_{1}^{2} U=0,
$$

$$
\begin{aligned}
& -\frac{1}{3}\left[(m-1) \partial_{1}{ }^{3}+3(m-2) \partial_{1} \partial_{2}\right] U+P \cdot \frac{1}{6}\left(\partial_{1}{ }^{3}+3 \partial_{1} \partial_{2}\right) U+\partial_{1} P \cdot \frac{1}{2} \partial_{1}{ }^{2} U=0, \\
& -\frac{1}{12}\left[(m-1)\left(\partial_{1}{ }^{4}+6 \partial_{1}{ }^{2} \partial_{2}\right)+(m-2)\left(4 \partial_{1} \partial_{3}+3 \partial_{2}{ }^{2}\right)\right] U \\
& +P \cdot \frac{1}{24}\left[\partial_{1}{ }^{4}+6 \partial_{1}{ }^{2} \partial_{2}+4 \partial_{1} \partial_{3}+3 \partial_{2}{ }^{2}\right] U+\partial_{1} P \cdot \frac{1}{6}\left[\partial_{1}{ }^{3}+3 \partial_{1} \partial_{2}\right] U+\frac{1}{2} \partial_{2} P \cdot \frac{1}{2} \partial_{1}{ }^{2} U=0 ;
\end{aligned}
$$

or reducing

$$
\begin{aligned}
P & =2(m-2), \\
\partial_{1} P & =\frac{2}{3} \frac{\partial_{1}{ }^{3} U}{\partial_{1}{ }^{2} U}, \\
\partial_{2} P & =\frac{1}{2} \frac{\left[\partial_{1}{ }^{4}+6 \partial_{1}{ }^{2} \partial_{2}\right] U}{\partial_{1}{ }^{2} U}-\frac{4}{9} \frac{\partial_{1}{ }^{3} U}{\partial_{1}{ }^{2} U} \frac{\left[\partial_{1}{ }^{3}+3 \partial_{1} \partial_{2}\right] U}{\partial_{1}{ }^{2} U},
\end{aligned}
$$

which are the conditions of a five-pointic contact: it is to be remarked that if only the first and second conditions are satisfied, we have a four-pointic contact, and if only the first condition is satisfied, a three-pointic contact.

9. We have to reduce the last-mentioned equations; suppose that $A, B, C$ are the first derived functions of $U$, then the equation $\partial_{1} U=0$ may be written

$$
A d x+B d y+C d z=0
$$


and this will be satisfied identically if

$$
\begin{aligned}
& d x=B \nu-C \mu, \\
& d y=C \lambda-A \nu, \\
& d z=A \mu-B \lambda,
\end{aligned}
$$

where $\lambda, \mu, \nu$ are arbitrary multipliers, which may be taken to be constants. We have therefore $\partial_{1}=\mathbf{D}$, where

$$
\mathbf{D}=(B \nu-C \mu) \partial_{x}+(C \lambda-A \nu) \partial_{y}+(A \mu-B \lambda) \partial_{z}
$$

10. The resulting expressions for $\partial_{1}{ }^{2} U, \partial_{1}{ }^{3} U, \partial_{1}{ }^{4} U$ may be exhibited in the reduced forms given by Hesse, viz. if $9=\lambda x+\mu y+\nu z$, we have

$$
\begin{aligned}
& \partial_{1}{ }^{2} U=P_{2} U-Q_{2} Q^{2}, \\
& \partial_{1}{ }^{3} U=P_{3} U-Q_{3} 9^{2}, \\
& \partial_{1}{ }^{4} U=P_{4} U-Q_{4} 9^{2},
\end{aligned}
$$

where the values of $P_{2}, P_{3}, P_{4} ; Q_{2}, Q_{3}, Q_{4}$ are as follows, viz. if $(a, b, c, f, g, h)$ are the second derived functions of $U$, and if

$$
H=\left|\begin{array}{lll}
a, & h, & g \\
h, & b, & f \\
g, & f, & c
\end{array}\right|
$$

be the Hessian; if, moreover,

$$
\Phi=-\left|\begin{array}{llll} 
& \lambda, & \mu, & \nu \\
\lambda & a, & h, & g \\
\mu & h, & b, & f \\
\nu & g, & f, & c
\end{array}\right|
$$

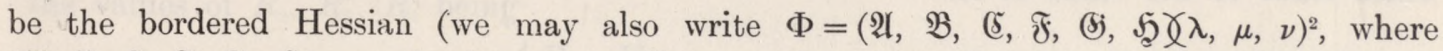
$\left(\mathfrak{A}, \mathfrak{B},(\mathfrak{S}, \mathfrak{F}, \mathfrak{S}, \mathfrak{H})\right.$ are the inverse coefficients of $(a, b, c, f, g, h)$, viz. $\mathfrak{A}=\left(b c-f^{2}\right)$, \&c.); and finally, if for shortness we write

then we have

$$
\begin{aligned}
& \Pi=\partial_{x} \Phi \cdot \partial_{\lambda} \Phi+\partial_{y} \Phi \cdot \partial_{\mu} \Phi+\partial_{z} \Phi \cdot \partial_{\nu} \Phi, \\
& \square=\partial_{x} H \cdot \partial_{\lambda} \Phi+\partial_{y} H \cdot \partial_{\mu} \Phi+\partial_{z} H \cdot \partial_{\nu} \Phi,
\end{aligned}
$$

$$
\begin{array}{ll}
P_{2}=\frac{m}{m-1} \Phi, & Q_{2}=\frac{1}{(m-1)^{2}} H, \\
P_{3}=\frac{m}{m-1} \mathbf{D} \Phi, & Q_{3}=\frac{1}{(m-1)^{2}} \mathbf{D} H, \\
P_{4}=\frac{m}{m-1} \mathbf{D}^{2} \Phi-\frac{m \Phi}{(m-1)^{2}} \Pi, & Q_{4}=\frac{1}{(m-1)^{2}} \mathbf{D}^{2} H-\frac{9}{(m-1)^{3}} \square+\frac{3(m-2)}{(m-1)^{3}} H \Phi . \\
27-2
\end{array}
$$


In the present case $U=0$, and we have

$$
\begin{aligned}
& \partial_{1}{ }^{2} U=-Q_{2} 9^{2}, \\
& \partial_{1}{ }^{3} U=-Q_{3} 9^{2}, \\
& \partial_{1}{ }^{4} U=-Q_{4} 9^{2} .
\end{aligned}
$$

11. Hence, substituting for $Q_{2}$ and $Q_{3}$ their values, the first and second of the equations for the five-pointic contact give

$$
\begin{aligned}
P & =2(m-2), \\
\partial_{1} P & =\frac{2}{3} \frac{\mathbf{D} H}{H},
\end{aligned}
$$

and observing that $\Pi$ is a linear function of $(X, Y, Z)$, and consequently that $P, \partial_{1} P$ denote simply the values which $I I$ assumes when $(x, y, z),(d x, d y, d z)$ are respectively substituted for $(X, Y, Z)$, we see at once that these two conditions will be satisfied if we put

$$
\Pi=\frac{2}{3} \frac{1}{H} D H+\Lambda . D U,
$$

where $\Lambda$ is an arbitrary constant, or, what is the same thing, an arbitrary function of $(x, y, z)$. We have thus the general equation of a conic of four-pointic contact.

12. The above value of $\Pi$ gives

$$
\partial_{2} P=\frac{2}{3} \frac{1}{H} \partial_{2} H+\Lambda \partial_{2} U,
$$

and the third equation of the system of conditions for a five-pointic contact is therefore

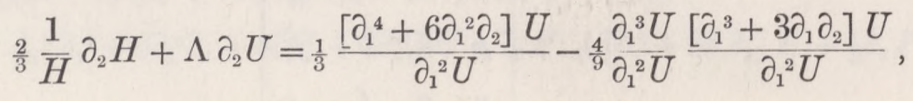

which leads to the value of $\Lambda$.

13. We have in general

$$
\begin{aligned}
\partial_{1}{ }^{2} U & =\frac{m}{m-1} \Phi U-\frac{1}{(m-1)^{2}} H 9^{2}, \\
\partial_{1}{ }^{3} U & =\frac{m}{m-1} \mathbf{D} \Phi \cdot U-\frac{1}{(m-1)^{2}} \mathbf{D} H \cdot 9^{2}, \\
\left(\partial_{1}{ }^{3}+3 \partial_{1} \partial_{2}\right) U & =\frac{m}{m-1} \mathbf{D} \Phi \cdot U-\frac{1}{(m-1)^{2}} \mathbf{D} H \cdot 9^{2}, \\
\left(\partial_{1}{ }^{4}+6 \partial_{1}{ }^{2} \partial_{2}\right) U & =\frac{m}{m-1}\left(2 \partial_{2} \Phi+\mathbf{D}^{2} \Phi+\frac{9}{m-1} \Pi\right) U \\
& \quad-\frac{1}{(m-1)^{2}}\left(2 \partial_{2} H+\mathbf{D}^{2} H+\frac{9}{m-1} \square-\frac{3(m-2)}{m-1} H \Phi\right) 9^{2},
\end{aligned}
$$


In the present case $U=0$, and we have

$$
\begin{aligned}
& \partial_{1}{ }^{2} U=-Q_{2} 9^{2}, \\
& \partial_{1}{ }^{3} U=-Q_{3} 9^{2}, \\
& \partial_{1}{ }^{4} U=-Q_{4} 9^{2} .
\end{aligned}
$$

11. Hence, substituting for $Q_{2}$ and $Q_{3}$ their values, the first and second of the equations for the five-pointic contact give

$$
\begin{aligned}
P & =2(m-2), \\
\partial_{1} P & =\frac{2}{3} \frac{\mathbf{D} H}{H},
\end{aligned}
$$

and observing that $\Pi$ is a linear function of $(X, Y, Z)$, and consequently that $P, \partial_{1} P$ denote simply the values which $\Pi$ II assumes when $(x, y, z),(d x, d y, d z)$ are respectively substituted for $(X, Y, Z)$, we see at once that these two conditions will be satisfied if we put

$$
\Pi=\frac{2}{3} \frac{1}{H} D H+\Lambda \cdot D U,
$$

where $\Lambda$ is an arbitrary constant, or, what is the same thing, an arbitrary function of $(x, y, z)$. We have thus the general equation of a conic of four-pointic contact.

12. The above value of $\Pi$ gives

$$
\partial_{2} P=\frac{2}{3} \frac{1}{H} \partial_{2} H+\Lambda \partial_{2} U
$$

and the third equation of the system of conditions for a five-pointic contact is therefore

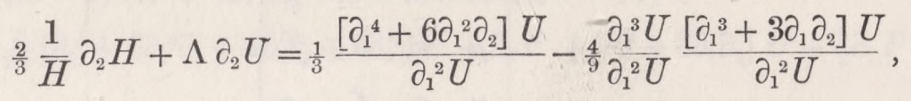

which leads to the value of $\Lambda$.

13. We have in general

$$
\begin{aligned}
\partial_{1}{ }^{2} U & =\frac{m}{m-1} \Phi U-\frac{1}{(m-1)^{2}} H 9^{2}, \\
\partial_{1}{ }^{3} U & =\frac{m}{m-1} \mathbf{D} \Phi \cdot U-\frac{1}{(m-1)^{2}} \mathbf{D} H \cdot \vartheta^{2}, \\
\left(\partial_{1}{ }^{3}+3 \partial_{1} \partial_{2}\right) U & =\frac{m}{m-1} \mathbf{D} \Phi \cdot U-\frac{1}{(m-1)^{2}} \mathbf{D} H \cdot 9^{2}, \\
\left(\partial_{1}{ }^{4}+6 \partial_{1}{ }^{2} \partial_{2}\right) U & =\frac{m}{m-1}\left(2 \partial_{2} \Phi+\mathbf{D}^{2} \Phi+\frac{9}{m-1} \Pi\right) U \\
& \quad-\frac{1}{(m-1)^{2}}\left(2 \partial_{2} H+\mathbf{D}^{2} H+\frac{9}{m-1} \square-\frac{3(m-2)}{m-1} H \Phi\right) \vartheta^{2},
\end{aligned}
$$


the last two of which have not yet been demonstrated. The value of $\partial_{2} H$ (which, however, is not required for the present purpose) is

$$
\partial_{2} H=\frac{-3(m-3)}{m-1} H \Phi+\frac{\frac{1}{2} \square}{m-1} 9,
$$

which also is not yet demonstrated.

14. Putting $U=0$, we have

$$
\begin{aligned}
\partial_{1}{ }^{2} U & =-\frac{1}{(m-1)^{2}} \quad H \quad 9^{2}, \\
\partial_{1}{ }^{3} U & =-\frac{1}{(m-1)^{2}} \quad \mathbf{D} H \cdot 9^{2}, \\
\left(\partial_{1}{ }^{3}+3 \partial_{1} \partial_{2}\right) U & =-\frac{1}{(m-1)^{2}} \quad \mathbf{D} H \cdot 9^{2}, \\
\left(\partial_{1}{ }^{4}+6 \partial_{1}{ }^{2} \partial_{2}\right) U & =-\frac{1}{(m-1)^{2}}\left(2 \partial_{2} H+\mathbf{D}^{2} H+\frac{9}{m-1} \square-\frac{3(m-2)}{m-1} H \Phi\right) ?^{2},
\end{aligned}
$$

and substituting,

$$
\begin{aligned}
& { }_{\frac{2}{3}}^{1} \partial^{\partial_{2} H}+\frac{1}{(m-1)^{2}} H 9^{2} \Lambda \\
& \quad=\frac{1}{3} \frac{1}{H}\left(2 \partial_{2} H+\mathbf{D}^{2} H+\frac{9}{m-1} \square-\frac{3(m-2)}{m-1} H \Phi\right)-\frac{4}{9}\left(\frac{\mathbf{D} H}{H}\right)^{2},
\end{aligned}
$$

where the term involving $\partial_{2} H$ disappears; the equation may be written

$$
\frac{9 H^{3} 9^{2}}{(m-1)^{2}} \Lambda=3 H\left(\mathbf{D}^{2} H+\frac{9}{m-1} \square-\frac{3(m-2)}{m-1} H \Phi\right)-4(\mathbf{D} H)^{2},
$$

which I will represent by

$$
\underset{(m-1)^{2}}{9 H^{3} 9^{2}} \Lambda=3 R_{2} R_{4}-4 R_{3}^{2}
$$

the values of $R_{2}, R_{3}, R_{\sharp}$ being

$$
\left\{\begin{array}{l}
R_{2}=H \\
R_{3}=\mathbf{D} H \\
R_{4}=\mathbf{D}^{2} H+\frac{9}{m-1} \square-\frac{3(m-2)}{m-1} H \Phi .
\end{array}\right.
$$

15. We have $R_{2}=H$, and it will be shown that

$$
\begin{aligned}
(m-1)^{2} R_{3}^{2}= & -9(m-2)^{2} H^{*} \Phi \\
& +3(m-2) H \square 9 \\
& -\quad \Psi 9^{2} \\
(m-1)^{2} R_{4}= & -12(m-2)^{2} H \Phi \\
& +4(m-2) \square 9 \\
& -\quad \Omega 9^{2},
\end{aligned}
$$


where for shortness

$$
\begin{aligned}
& \left.\Psi=(\mathfrak{A}, \mathfrak{B}, \mathfrak{f}, \mathfrak{F}, \mathfrak{S}, \mathfrak{S}) \partial_{x} H, \partial_{y} H, \partial_{z} H\right)^{2}, \\
& \Omega=\left(\mathfrak{A}, \mathfrak{B}, \mathfrak{(}, \mathfrak{F}, \mathfrak{S}, \mathfrak{S} \nvdash \partial_{x}, \partial_{y}, \partial_{z}\right)^{2} H,
\end{aligned}
$$

and hence, writing

$$
9 H^{3} 9^{2} \Lambda=3 R_{2}(m-1)^{2} R_{4}-4(m-1)^{2} R_{3}^{2},
$$

we have

$$
9 H^{3} \Lambda=-3 \Omega H+4 \Psi
$$

or replacing $\Omega, \Psi$ by their values,

$$
\Lambda=\frac{1}{9 H^{3}}\left\{\begin{array}{l}
-3\left(\mathfrak{A}, \mathfrak{B}, \mathfrak{C}, \mathfrak{F}, \mathfrak{S}, \mathfrak{S} \backslash \partial_{x}, \partial_{y}, \partial_{z}\right)^{2} H \cdot H \\
+4\left(\mathfrak{A}, \mathfrak{B}, \mathfrak{C}, \mathfrak{F}, \mathfrak{E S}, \mathfrak{S} \backslash \partial_{x} H, \partial_{y} H, \partial_{z} H\right)^{2}
\end{array}\right\},
$$

and $\Lambda$ having this value, the equation of the five-pointic conic is

$$
D^{2} U-\left(\frac{2}{3} \frac{1}{H} D H+\Lambda D U\right) D U=0,
$$

where it will be recollected that the current coordinates are $(X, Y, Z)$, and that $D$ denotes $X \partial_{x}+Y \partial_{y}+Z \partial_{z}$.

16. I remark, in passing, that the problem of finding the circle of curvature at a given point of a plane curve, is in fact that of determining the conic having with the curve at the given point a three-pointic contact, and besides passing through two given points. The equation of a conic having an ordinary contact, is

$$
D^{2} U-\Pi D U=0,
$$

where

$$
\Pi=a X+b Y+c Z
$$

and the condition of a three-pointic contact is

$$
a x+b y+c z=2(m-2) \text {. }
$$

Let the coordinates of the two given points be

$$
\left(x_{1}, y_{1}, z_{1}\right), \quad\left(x_{2}, y_{2}, z_{2}\right),
$$

and let $\left(D^{2} U\right)_{1} \&$ c. be the corresponding values of $D^{2} U$, \&c., then we have

$$
\begin{aligned}
& a X+b Y+c Z=\frac{D^{2} U}{D U}, \\
& a x_{1}+b y_{1}+c z_{1}=\left(\begin{array}{c}
D^{2} U \\
\overline{D U}
\end{array}\right)_{1}, \\
& a x_{2}+b y_{2}+c z_{2}=\left(\frac{D^{2} U}{D U}\right)_{2} ;
\end{aligned}
$$


and if from the four equations we eliminate $a, b, c$, we find

$$
\left|\begin{array}{cccl}
X D U, & Y D U, & Z D U, & D^{2} U \\
x, & y, & z, & 2(m-2) \\
x_{1}, & y_{1}, & z_{1}, & \left(\begin{array}{l}
D^{2} U \\
D U
\end{array}\right)_{1} \\
x_{2}, & y_{2}, & z_{2}, & \left(\begin{array}{l}
D^{2} U \\
D U
\end{array}\right)_{2}
\end{array}\right|=0
$$

for the equation of the conic in question; $x, y, z$ being the coordinates of the point of contact, and $X, Y, Z$ current coordinates.

\section{Demonstration of Identities assumed in the preceding section.}

Proof of the expressions for $\left(\partial_{1}^{3}+3 \partial_{1} \partial_{2}\right) U$ and $\left(\partial_{1}^{4}+6 \partial_{1}^{2} \partial_{2}\right) U$ :

17. It will be remembered that $\partial_{1}, \partial_{2}$ stand originally for

$$
\begin{aligned}
& d x \partial_{x}+d y \partial_{y}+d z \partial_{z}, \\
& d^{2} x \partial_{x}+d^{2} y \partial_{y}+d^{2} z \partial_{z},
\end{aligned}
$$

and that $A, B, C$ being the first derived functions of $U, d x, d y, d z$ are changed into

$$
B \nu-C \mu, \quad C \lambda-A \nu, \quad A \mu-B \lambda,
$$

and that the resulting value of $\partial_{1}$, viz.

$$
(B \nu-C \mu) \partial_{x}+(C \lambda-A \nu) \partial_{y}+(A \mu-B \lambda) \partial_{z}
$$

is also represented by $\mathbf{D}$, so that $\partial_{1}=\mathbf{D}$. The corresponding values of $d^{2} x, d^{2} y, d^{2} z$ are

$$
\begin{aligned}
& d^{2} x=\nu d B-\mu d C \\
& d^{2} y=\lambda d C-\nu d A \\
& d^{2} z=\mu d A-\lambda d B
\end{aligned}
$$

where we have

$$
d A=a d x+h d y+g d z, \& c .
$$

in which $d x, d y, d z$ are to be replaced by the values

$$
B \nu-C \mu, \quad C \lambda-A \nu, \quad A \mu-B \lambda
$$

and $\partial_{2}$ really denotes what

$$
d^{2} x \partial_{x}+d^{2} y \partial_{y}+d^{2} z \partial_{z}
$$

becomes when the above values are substituted for $d^{2} x, d^{2} y, d^{2} z$. But in the expressions $\partial_{1}{ }^{2} U, \partial_{1} \partial_{2} U$ \&c., the symbols $\partial_{x}, \partial_{y}, \partial_{z}$ contained in $\partial_{1}$ and $\partial_{2}$ operate only on $U$, and not on the variable quantities $A, B, C$, \&c. contained in $\partial_{1}$ and $\partial_{2}$. 
18. If now we treat $\partial_{1}$ as an operand, that is, perform the differentiations on the variable quantities $A, B, C$ which enter into $\partial_{1}$, we obtain

$$
\partial_{1} \cdot \partial_{1}=\partial_{2}
$$

or, what is the same thing, operating on $\partial_{1} U$ with $\partial_{1}$, the result is

and in like manner

$$
\partial_{1} \cdot \partial_{1} U=\partial_{1}^{2} U+\partial_{2} U=\left(\partial_{1}^{2}+\partial_{2}\right) U
$$

$$
\begin{aligned}
& \partial_{1} \cdot \partial_{1}^{2} U=\left(\partial_{1}^{3}+2 \partial_{1} \partial_{2}\right) U \\
& \partial_{1} \cdot \partial_{1}^{3} U=\left(\partial_{1}^{4}+3 \partial_{1}{ }^{2} \partial_{2}\right) U .
\end{aligned}
$$

It is, in fact, upon these principles that Hesse's values of $\partial_{1}^{2} U, \partial_{1}{ }^{3} U$, \&c. were obtained, and we may by means of them obtain the other expressions assumed in the preceding section.

19. In fact, starting from Hesse's equation,

$$
\partial_{1}^{2} U=\frac{m}{m-1} \Phi U-\frac{1}{(m-1)^{2}} H 9^{2}
$$

we have

$$
\left(\partial_{1}{ }^{3}+2 \partial_{1} \partial_{2}\right) U=\frac{m}{m-1}(U \mathbf{D} \Phi+\Phi \mathbf{D} U)-\frac{1}{(m-1)^{2}}\left(\mathbf{D} H \cdot \Upsilon^{2}+H .29 \mathbf{D}\right)
$$

But we have identically $\mathbf{D} U=0, \mathbf{D}=0$, and this equation becomes therefore

$$
\left(\partial_{1}{ }^{3}+2 \partial_{1} \partial_{2}\right) U=\frac{m}{m-1} U \mathbf{D} \Phi-\frac{1}{(m-1)^{2}} \mathbf{D} H \cdot \stackrel{9}{ }^{2}
$$

this is precisely Hesse's value of $\partial_{1}{ }^{3} U$, and thus we have $\partial_{1} \partial_{2} U=0$, and therefore

$$
\left(\partial_{1}{ }^{3}+3 \partial_{1} \partial_{2}\right) U=\partial_{1}{ }^{3} U=\frac{m}{m-1} U \mathbf{D} \Phi-\frac{1}{(m-1)^{2}} \mathbf{D} H .9^{2}
$$

20. In like manner, starting from the expression of $\partial_{1}{ }^{3} U$, we have

$$
\left(\partial_{1}{ }^{4}+3 \partial_{1}{ }^{2} \partial_{2}\right) U=\frac{m}{m-1}(\mathbf{D} U \cdot \mathbf{D} \Phi+U \mathbf{D} \cdot \mathbf{D} \Phi)-\frac{1}{(m-1)^{2}}\left(\mathbf{D} H \cdot 2 \Re \mathbf{D}+\rightarrow^{2} \mathbf{D} \cdot \mathbf{D} H\right)
$$

or since $\mathbf{D} U$ and $\mathbf{D} 9$ vanish identically, and the values of $\mathbf{D} . \mathbf{D} \Phi, \mathbf{D} . \mathbf{D} H$ are $\partial_{2} \Phi+\mathbf{D}^{2} \Phi, \partial_{2} H+\mathbf{D}^{2} H$, we have

$$
\left(\partial_{1}{ }^{4}+3 \partial_{1}{ }^{2} \partial_{2}\right) U=\frac{m}{m-1} U\left(\partial_{2} \Phi+\mathbf{D}^{2} \Phi\right)-\frac{1}{(m-1)^{2}} 9^{2}\left(\partial_{2} H+\mathbf{D}^{2} H\right)
$$

and if from the double of this equation we subtract Hesse's equation,

$$
\partial_{1}^{4} U=\frac{m}{m-1} U\left(\mathbf{D}^{2} \Phi-\frac{9}{m-1} \Pi\right)-\frac{1}{(m-1)^{2}} 9^{2}\left(\mathbf{D}^{2} H-\frac{9 \square}{m-1}+\frac{3(m-2)}{m-1} H \Phi\right)
$$


we find the required relation,

$$
\begin{aligned}
\left(\partial_{1}{ }^{4}+6 \partial_{1}{ }^{2} \partial_{2}\right) U= & \frac{m}{m-1}\left(2 \partial_{2} \Phi+\mathbf{D}^{2} \Phi+\frac{\vartheta}{m-1} \Pi\right) U \\
& \quad-\frac{1}{(m-1)^{2}}\left(2 \partial_{2} H+\mathbf{D}^{2} H+\frac{2 \square}{m-1}-\frac{3(m-2)}{m-1} H \Phi\right) 9^{2} .
\end{aligned}
$$

Proof of the expression for $\partial_{2} H$ :

21. We have

where

$$
\partial_{2} H=\partial_{x} H(\nu d B-\mu d C)+\partial_{y} H(\lambda d \dot{C}-\nu d B)+\partial_{z} H(\mu d A-\lambda d B)
$$

$$
\begin{aligned}
& d A=a d x+h d y+g d z, \\
& d B=h d x+b d y+f d z, \\
& d C=g d x+f d y+c d z,
\end{aligned}
$$

in which $d x, d y, d z$ are to be replaced by their values: we have therefore

$$
\begin{aligned}
\partial_{2} H=\partial_{x} H & \{\nu[(b C-f B) \lambda+(f A-h C) \mu+(h B-b A) \nu] \\
& -\mu[(f C-c B) \lambda+(c A-g C) \mu+(g B-f A) \nu]\}, \\
+ & \text { \&c. }
\end{aligned}
$$

where the coefficient of $\partial_{x} H$ is

$$
0 \lambda^{2}+(g C-c A) \mu^{2}+(h B-b A) \nu^{2}+(2 f A-g B-h C) \mu \nu+(b C-f B) \nu \lambda+(c B-f C) \lambda \mu
$$

or, since we have

$$
\begin{aligned}
& (m-1) A=a x+h y+g z, \\
& (m-1) B=h x+b y+f z, \\
& (m-1) C=g x+f y+c z,
\end{aligned}
$$

the coefficient, omitting the factor $\frac{1}{m-1}$, which will be afterwards restored, is

$$
\begin{aligned}
& 0 \lambda^{2} \\
& +[g(g x+f y+c z)-c(a x+h y+g z)] \mu^{2} \\
& +[h(h x+b y+f z)-b(a x+h y+g z)] \nu^{2} \\
& +[2 f(a x+h y+g z)-g(h x+b y+f z)-h(g x+f y+c z)] \mu \nu \\
& +[b(g x+f y+c z)-f(h x+b y+f z)] \nu \lambda \\
& +[c(h x+f y+c z)-f(g x+f y+c z)] \lambda \mu \\
& =0 \lambda^{2} \\
& +(-\mathfrak{B} x+\mathfrak{S} y) \mu^{2} \\
& +\left(-(5 x+\sqrt{S} z) \nu^{2}\right. \\
& +(-2 \mathfrak{F} x+(\mathfrak{S} y+\mathfrak{S} z) \mu \nu \\
& +(-(5) x+\quad \mathfrak{2} z) \nu \lambda \\
& +(-\mathfrak{S} x+\mathfrak{P} y \quad) \lambda \mu \text {, }
\end{aligned}
$$

C. IV. 
which is equal to

$$
\begin{aligned}
& -\left(\mathfrak{A} \lambda^{2}+\mathfrak{B} \mu^{2}+\left(\mathfrak{C} \nu^{2}+2 \mathfrak{F} \mu \nu+2 \mathfrak{S} \nu \lambda+2 \mathfrak{S} \lambda \mu\right) x\right. \\
& +(\mathfrak{2} \lambda+\mathfrak{S} \mu+\mathfrak{S} \nu)(\lambda x+\mu y+\nu z) \text {. }
\end{aligned}
$$

The coefficients of $\partial_{y} H, \partial H_{z}$ have a similar form; and uniting the three terms, observing that $x \partial_{x} H+y \partial_{y} H+z \partial_{z} H$ is equal to $3(m-2) H$, and attending to the values of $\Phi, \square, 9$, we have, restoring the omitted factor $\frac{1}{m-1}$,

$$
\partial_{2} H=\frac{-3(m-2)}{m-1} H \Phi+\frac{\frac{1}{2} \square}{m-1} 9 .
$$

Proof of the expressions for $(\mathbf{D} H)^{2}$ and $\mathbf{D}^{2} H$ :

22. These are obtained (for the particular case $m=4$, which makes but little difference) in Mr Salmon's Higher Plane Curves, pp. 88 and 89, and I merely reproduce his investigation; we have

$$
(\mathbf{D} H)^{2}=\left\{(B \nu-C \mu) \partial_{x} H+(C \lambda-A \nu) \partial_{y} H+(A \mu-B \lambda) \partial_{z} H\right\}^{2},
$$

or, what is the same thing,

$$
(\mathbf{D} H)^{2}=\left\{\lambda\left(C \partial_{y}-B \partial_{z}\right) H+\mu\left(A \partial_{z}-C \partial_{x}\right) H+\nu\left(B \partial_{x}-A \partial_{z}\right) H\right\}^{2} ;
$$

and if we consider first the term which contains $\lambda^{2}$, the coefficient is

$$
\left\{\left(C \partial_{y}-B \partial_{z}\right) H\right\}^{2}
$$

Now making use of the equations

and

$$
\begin{aligned}
& (m-1) A=a x+h y+g z, \\
& (m-1) B=h x+b y+f z, \\
& (m-1) C=g x+f y+c z,
\end{aligned}
$$

we have

$$
m(m-1) U=a x^{2}+b y^{2}+c z^{2}+2 f y z+2 g z x+2 h x y=0,
$$

$$
\begin{aligned}
(m-1)^{2} \quad C^{2} & =(g x+f y+c z)^{2}-c\left(a x^{2}+b y^{2}+c z^{2}+2 f y z+2 g z x+2 h x y\right) \\
& =-\mathfrak{B} x^{2}+2 \mathfrak{S} x y-\mathfrak{A} y^{2} \\
(m-1)^{2} B C & =(h x+b y+f z)(g x+f y+c z)-f\left(a x^{2}+b y^{2}+c z^{2}+2 f y z+2 g z x+2 h x y\right) \\
& =\mathfrak{F} x^{2}-\mathfrak{B} x y-\mathfrak{S} x z+\mathfrak{A} y z \\
(m-1)^{2} B^{2} & =(h x+b y+f z)^{2}-b\left(a x^{2}+b y^{2}+c z^{2}+2 f y z+2 g z x+2 h x y\right) \\
& =-\left(5 x^{2}+2\left(\mathfrak{S} x z-\mathfrak{A} z^{2}\right.\right.
\end{aligned}
$$

and hence

$$
\begin{aligned}
(m-1)^{2}\left(\left(C \partial_{y}-B \partial_{z}\right) H\right)^{2}= & \left(-\mathfrak{B} x^{2}+2 \mathfrak{S} x y-\mathfrak{A} y^{2}\right)\left(\partial_{y} H\right)^{2} \\
+ & 2\left(-\mathfrak{F} x^{2}+\mathfrak{S} x y+\mathfrak{S} x z-\mathfrak{A} y z\right) \partial_{y} H . \partial_{z} H \\
& +\left(-\left(\sqrt{5} x^{2}+2 \mathfrak{S} x z-\mathfrak{A} z^{2}\right)\left(\partial_{z} H\right)^{2}\right. \\
= & -x^{2}\left(\mathfrak{B}, \mathfrak{F},\left(\mathfrak{5} \gamma \partial_{y} H, \partial_{z} H\right)^{2}\right. \\
& +2 x\left(y \partial_{y} H+z \partial_{z} H\right)\left(\mathfrak{S} \partial_{y} H+\left(\mathfrak{S} \partial_{z} H\right)\right. \\
& -\mathfrak{A}\left(y \partial_{y} H+z \partial_{z} H\right)^{2},
\end{aligned}
$$


and

so that the term is

$$
y \partial_{y} H+z \partial_{z} H=3(m-2) H-z \partial_{z} H
$$$$
\text { - } x^{2}\left(\mathfrak{B}, \mathfrak{F},(\mathfrak{5}) \partial_{y} H, \partial_{x} H\right)^{2}
$$$$
+2 x\left(3(m-2) H-z \partial_{z} H\right)\left(\mathfrak{S} \partial_{y} H+(5) \partial_{z} H\right)
$$$$
-\mathfrak{A}\left(3(m-2) H-z \partial_{z} H\right)^{2} \text {; }
$$

or, reducing, we have

$$
\begin{aligned}
(m-1)^{2}\left\{\left(C \partial_{y}-B \partial_{z}\right) H\right\}^{2} & \\
= & -\quad x^{2}\left(\mathfrak{A}, \mathfrak{B}, \mathfrak{C}, \mathfrak{F}, \mathfrak{F}, \mathfrak{S} \nvdash \partial_{x} H, \partial_{y} H, \partial_{z} H\right)^{2} \\
& +6(m-2) H x\left(\mathfrak{H} \partial_{x} H+\mathfrak{H} \partial_{y} H+\mathfrak{S} \partial_{z} H\right) \\
& -9(m-2)^{2} \mathfrak{A} H^{2} .
\end{aligned}
$$

23. The other terms may be obtained in a similar manner; and it is easy to see that, collecting all the terms, the sum will be

$$
-(\lambda x+\mu y+\nu z)^{2}\left(\mathfrak{A}, \mathfrak{B}, \mathfrak{(}, \mathfrak{F}, \mathfrak{S}, \mathfrak{S}\left\{\partial_{x} H, \partial_{y} H, \partial_{z} H\right)^{2}\right.
$$

$+6(m-2) H(\lambda x+\mu y+\nu 2)\left\{(\mathfrak{A} \lambda+\mathfrak{S} \mu+\mathfrak{E} \nu) \partial_{x} H+(\mathfrak{S} \lambda+\mathfrak{B} \mu+\mathfrak{\mho} \nu) \partial_{y} H+\left(\mathfrak{S} \lambda+\mathfrak{\mho} \mu+(\mathfrak{C} \nu) \partial_{z} H\right\}\right.$

$$
-9(m-2)^{2} H^{2}(\mathfrak{A}, \mathfrak{B}, \mathfrak{(}, \mathfrak{F}, \mathfrak{S}, \mathfrak{S} \gamma \lambda, \mu, \nu)^{2} \text {; }
$$

or, attending to the signification of the symbols $9, \Phi, \square, \Psi$, we have

$$
\begin{aligned}
(m-1)^{2}(\mathbf{D} H)^{2}= & -9(m-2)^{2} H^{2} \Phi \\
& +3(m-2) H \square 9 \\
& -\Psi 9^{2} .
\end{aligned}
$$

24. Next

$$
\mathbf{D}^{2} H=\left((B \nu-C \mu) \partial_{x}+(C \lambda-A \nu) \partial_{y}+(A \mu-B \lambda) \partial_{z}\right)^{2} H,
$$

or, what is the same thing,

$$
\mathbf{D}^{2} H=\left\{\lambda\left(C \partial_{y}-B \partial_{z}\right)+\mu\left(A \partial_{z}-C \partial_{x}\right)+\nu\left(B \partial_{x}-A \partial_{z}\right)\right\}^{2} H
$$

and if we attend first to the term which contains $\lambda^{2}$, the coefficient is

$$
\left(C \partial_{y}-B \partial_{z}\right)^{2} H \text {. }
$$

Now substituting for $C^{2}, C B, B^{2}$ as before, we have

$$
\begin{aligned}
& (m-1)^{2}\left(C \partial_{y}-B \partial_{z}\right)^{2} H=\left(-\mathfrak{B} x^{2}+2 \mathfrak{S} x y \quad-\mathfrak{2}\left(y^{2}\right) \partial_{y}{ }^{2} H\right. \\
& +2\left(-\mathfrak{F} x^{2}+\mathfrak{F} x y+\mathfrak{S} x z-\mathfrak{A} y z\right) \partial_{y} \partial_{z} H \\
& +\left(-\left(5 x^{2}+25 x z \quad-2\left(z^{2}\right) \partial_{z}{ }^{2} H\right.\right. \\
& =-x^{2}\left(\mathfrak{B}, \tilde{\mho},(\mathfrak{E}) \partial_{y}, \partial_{z}\right)^{2} H \\
& +2 x\left(y \partial_{y}+z \partial_{z}\right)\left(\mathfrak{S} \partial_{y}+\left(\mathfrak{S} \partial_{z}\right) H\right. \\
& -\mathfrak{A}\left(y \partial_{y}+z \partial_{z}\right)^{2} H \text {, }
\end{aligned}
$$


where it is to be observed that the symbols of differentiation affect $H$ only. We have

$$
\begin{aligned}
\left(y \partial_{y}+z \partial_{z}\right)^{2} H= & \left(x \partial_{x}+y \partial_{y}+z \partial_{z}\right)^{2} H \\
& -2\left(x \partial_{x}+y \partial_{y}+z \partial_{z}\right) x \partial_{x} H \\
& +\left(x \partial_{x}\right)^{2} H
\end{aligned}
$$

where $H$ is a homogeneous function of the degree $3 m-6 ; x \partial_{x} H$, since the $x$ is not affected by the differentiation, must be treated as of the degree $3 m-7$, and $\left(x \partial_{x}\right)^{2} H$, for the like reason, stands for $x^{2} \partial_{x}^{2} H$; we have therefore

$$
\begin{aligned}
\left(y \partial_{y}+z \partial_{z}\right)^{2} H= & (3 m-6)(3 m-7) H \\
& -2(3 m-7) x \partial_{x} H \\
& +x^{2} \partial_{x}{ }^{2} H .
\end{aligned}
$$

In like manner,

$$
\begin{aligned}
\left(y \partial_{y}+z \partial_{z}\right)\left(\mathfrak{S} \partial_{y}+\mathfrak{S} \partial_{z}\right) H & =\left(x \partial_{x}+y \partial_{y}+z \partial_{z}\right)\left(\mathfrak{S} \partial_{y}+\mathfrak{S} \partial_{z}\right) H-x \partial_{x}\left(\mathfrak{S} \partial_{y}+\mathfrak{S} \partial_{z}\right) H \\
& =(3 m-7)\left(\mathfrak{S} \partial_{y}+\mathfrak{S} \partial_{z}\right) H-x \partial_{x}\left(\mathfrak{S} \partial_{y}+\mathfrak{S} \partial_{z}\right) H
\end{aligned}
$$

and hence

$$
\begin{aligned}
(m-1)^{2}\left(C \partial_{y}-B \partial_{z}\right)^{2} H= & -x^{2}(\mathfrak{B}, \mathfrak{F}, \mathfrak{\complement})\left(\partial_{y}, \partial_{z}\right)^{2} H \\
& +2 x\left[(3 m-7)\left(\mathfrak{S} \partial_{y}+\mathfrak{S} \partial_{z}\right) H-x \partial_{x}\left(\mathfrak{S} \partial_{y}+\mathfrak{S} \partial_{z}\right) H\right] \\
& -\mathfrak{A}\left[(3 m-6)(3 m-7) H-2(3 m-7) x \partial_{x} H+x^{2} \partial_{x}{ }^{2} H\right] \\
= & -x^{2}\left(\mathfrak{A}, \mathfrak{B}, \mathfrak{E}, \mathfrak{F}, \mathfrak{S}, \mathfrak{S}\left(\partial_{x}, \partial_{y}, \partial_{z}\right)^{2} H\right. \\
& +2(3 m-7) x\left(\mathfrak{A} \partial_{x}+\mathfrak{S} \partial_{y}+\left(\mathfrak{S} \partial_{z}\right) H\right. \\
& -(3 m-6)(3 m-7) \mathfrak{A} H .
\end{aligned}
$$

25. The other terms are formed in a similar manner; and collecting all the terms, we have

$$
\begin{aligned}
& (m-1)^{2} \mathbf{D}^{2} H=-(3 m-6)(3 m-7)\left(\mathfrak{A}, \mathfrak{B}, \quad(\mathfrak{F} \quad \mathfrak{F}, \quad \mathfrak{S}, \mathfrak{S})(\lambda, \mu, \nu)^{2} H\right. \\
& +2(3 m-7)(\lambda x+\mu y+\nu z)\left(\left(\mathfrak{A} \lambda+\mathfrak{S} \mu+(\mathfrak{S} \nu) \partial_{x} H+(\mathfrak{S} \lambda+\mathfrak{B} \mu+\sqrt{\mho} \nu) \partial_{y} H+\left(\mathfrak{S} \lambda+\mathfrak{F} \mu+(\mathfrak{5} \nu) \partial_{z} H\right)\right.\right. \\
& -(\lambda x+\mu y+\nu z)^{2}\left(\mathfrak{A}, \mathfrak{B},\left(\mathfrak{5}, \mathfrak{F}, \mathbb{S}, \mathfrak{S} \gamma \partial_{x}, \partial_{y}, \partial_{z}\right)^{2} H,\right.
\end{aligned}
$$

or, attending to the signification of the symbols $9, \Phi, \square, \Omega$, this is

$$
\begin{gathered}
(m-1)^{2} \mathbf{D}^{2} H=-(3 m-6)(3 m-7) H \Phi \\
+(3 m-7) 9 \square \\
-\Omega 9^{2} .
\end{gathered}
$$


Proof of the expressions for $(m-1)^{2} R_{3}{ }^{2},(m-1)^{2} R_{4}$ :

26. We have

$$
\begin{aligned}
(m-1)^{2} R_{3}{ }^{2}= & (m-1)^{2}(\mathbf{D} H)^{2} \\
= & -9(m-2)^{2} H^{2} \Phi \\
& +3(m-2) H \square 9 \\
& -\Psi 9^{2}, \\
(m-1)^{2} R_{4}= & (m-1)^{2} \mathbf{D}^{2} H+(m-1) 9 \square-(3 m-6)(m-1) \Phi H \\
= & -12(m-2)^{2} H \Phi \\
& +4(m-2) \square 9 \\
& -\Omega 9^{2},
\end{aligned}
$$

which are the expressions required.

Proof of corresponding expressions for $(m-1)^{6} Q_{3}{ }^{2},(m-1)^{4} Q_{4}$ :

27. We have

$$
\begin{aligned}
(m-1)^{6} Q_{3}{ }^{2}= & (m-1)^{2}(\mathbf{D} H)^{2} \\
= & -9(m-2)^{2} H^{2} \Phi \\
& +3(m-2) H \square \Phi \\
& -\Psi 9, \\
(m-1)^{4} Q_{4}= & (m-1)^{2} \mathbf{D}^{2} H-(m-1) 9 \square+(3 m-6)(m-1) H \Phi \\
= & -6(m-2)(m-3) H \Phi \\
& +2(m-3) \square 9 \\
& -\Omega 9^{2} ;
\end{aligned}
$$

to which I join

$$
(m-1)^{2} Q_{2}=H .
$$

28. We have consequently

$$
\begin{aligned}
(m-1)^{6}\left(3 Q_{2} Q_{4}-Q_{3}{ }^{2}\right)= & -9(m-2)(m-4) H^{2} \Phi \\
& +3(m-4) H \square Я \\
& +(-3 \Omega H+\Psi) \stackrel{2}{2},^{2}
\end{aligned}
$$

and

$$
\left.(m-1)^{6}\left(3(m-2) Q_{2} Q_{4}-2(m-3) Q_{3}{ }^{2}\right)=(-3(m-2) \Omega H+2(m-3) \Psi)\right)^{2},
$$

which for $m=4$ becomes

$$
729\left(3 Q_{2} Q_{4}-Q_{3}{ }^{2}\right)=(-3 \Omega H+\Psi) 9^{2} .
$$

29. In the case $m=4$, we have Hesse's theorem, that the equation $3 Q_{2} Q_{4}-Q_{3}=0$ gives a curve of the 14th order, which passes through the points of contact of the double tangents, viz. substituting for $\Omega, \Psi$ their values, the equation of this curve is

$$
\begin{aligned}
& -3 H\left(\mathfrak{A}, \mathfrak{B}, \mathfrak{C}, \mathfrak{F}, \mathfrak{S}, \mathfrak{H} \gamma \partial_{x}, \partial_{y}, \partial_{z}\right)^{2} H \\
& +\quad\left(\mathfrak{A}, \mathfrak{B}, \mathfrak{5}, \mathfrak{F}, \mathfrak{S}, \mathfrak{S} \gamma_{x} H, \partial_{y} H, \partial_{z} H\right)^{2}=0 .
\end{aligned}
$$


I have added these remarks for the sake of pointing out the striking resemblance of the expressions which occur in the double-tangent problem for $m=4$, and in the present theory of the five-pointic conic for any value whatever of $m$. It has not hitherto been shown what the expressions $3(m-2) Q_{3} Q_{4}-2(m-3) Q_{3}{ }^{2}$ and $-3(m-2) \Omega H+2(m-3) \Psi$ respectively denote, except in the particular case $m=4$.

\section{Application of the Formula to the Cubic.}

30. I shall apply the formula for the five-pointic conic to a cubic; to avoid confusion as to a numerical factor, I write $U^{\prime}, H^{\prime}$ in the place of $U, H$, so that we have

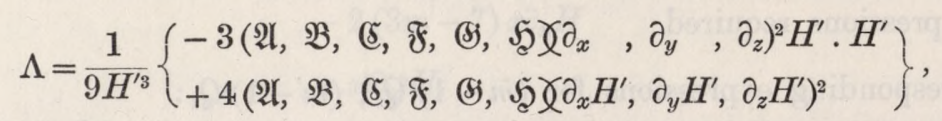

and then the equation of the five-pointic conic is

$$
D^{2} U^{\prime}-\frac{2}{3}\left(\frac{1}{H^{\prime}} D H^{\prime}+\Lambda D U^{\prime}\right) D U^{\prime}=0 .
$$

I take as the equation of the cubic,

$$
U=x^{3}+y^{3}+z^{3}+6 l x y z=0 ;
$$

the formulæ Table No. 70 of my Third Memoir on Quantics, Phil. Trans., vol. cxlvI. (1856), pp. 627-647, [144], putting $H$ for $H U$, give

Hence writing

$$
H=l^{2}\left(x^{3}+y^{3}+z^{3}\right)-\left(1+2 l^{3}\right) x y z .
$$

$$
U^{\prime}=\frac{1}{6}\left(x^{3}+y^{3}+z^{3}+6 l x y z\right)
$$

the first derived functions are

$$
\frac{1}{2}\left(x^{2}+2 l y z\right), \quad \frac{1}{2}\left(y^{2}+2 l z x\right), \quad \frac{1}{2}\left(z^{2}+2 l x y\right) ;
$$

the second derived functions, or $(a, b, c, f, g, h)$, are $(x, y, z, l x, l y, l z)$, whence $H^{\prime}=-H$; the inverse coefficients $(\mathfrak{A}, \mathfrak{B}, \mathfrak{C}, \mathfrak{F}, \mathfrak{F}, \mathfrak{S})$ are

$$
\left(y z-l^{2} x^{2}, z x-l^{2} y^{2}, x y-l^{2} z^{2}, l^{2} y z-l x^{2}, l^{2} z x-l y^{2}, l^{2} x y-l z^{2}\right) \text {; }
$$

and putting $U^{\prime}=\frac{1}{6} U$ and $H^{\prime}=-H$, we have

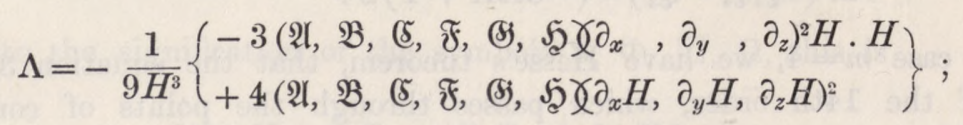

and the equation of the five-pointic conic is

$$
D^{2} U-\left(\frac{2}{3} \frac{1}{H} D H+\frac{1}{6} \Lambda D U\right) D U=0 .
$$


31. We have

which is

( $\mathfrak{A}, \mathfrak{B},\left(\mathfrak{S}, \mathfrak{F}, \mathfrak{S}, \mathfrak{j} \gamma \partial_{x}, \partial_{y}, \partial_{z}\right)^{2} H$

$$
\begin{aligned}
= & \left(y z-l^{2} x^{2}\right) \cdot 6 l^{2} x \\
+ & \left(z x-l^{2} y^{2}\right) \cdot 6 l^{2} y \\
+ & \left(x y-l^{2} z^{2}\right) \cdot 6 l^{2} z \\
+ & 2\left(l^{2} y z-l x^{2}\right) \cdot-\left(1+2 l^{3}\right) a \\
+ & 2\left(l^{2} z x-l y^{2}\right) \cdot-\left(1+2 l^{3}\right) y \\
+ & 2\left(l^{2} x y-l z^{2}\right) \cdot-\left(1+2 l^{3}\right) z
\end{aligned}
$$

or we have

$$
\begin{aligned}
= & 18 l^{2} x y z-6 l^{4}\left(x^{3}+y^{3}+z^{3}\right) \\
& -6 l^{2}\left(1+2 l^{3}\right) x y z+2 l\left(1+2 l^{3}\right)\left(x^{3}+y^{3}+z^{3}\right), \\
= & \left(12 l^{2}-12 l^{5}\right) x y z+\left(2 l-2 l^{4}\right)\left(x^{3}+y^{3}+z^{3}\right), \\
= & 2\left(l-l^{4}\right)\left(x^{3}+y^{3}+z^{3}+6 l x y z\right),
\end{aligned}
$$

(ㄱ, $\left.\mathfrak{B}, \mathfrak{C}, \mathfrak{F}, \mathfrak{S}, \mathfrak{S} \chi \lambda_{*}, \partial_{y}, \partial_{z}\right)^{2} H=-2 S . U$,

where $S$ is the quartinvariant (see the Table No. 70). For the present purpose $U=0$, and consequently

(ㄱ, $\left.\mathfrak{B}, \mathfrak{C}, \mathfrak{F}, \mathfrak{S}, \mathfrak{S} \zeta \partial_{x}, \partial_{y}, \partial_{z}\right)^{2} H=0$.

32. Next,

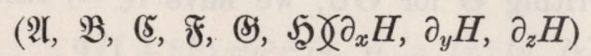

$$
\begin{aligned}
= & \left(y z-l^{2} x^{2}\right)\left[3 l^{2} x^{2}-\left(1+2 l^{3}\right) y z\right]^{2} \\
& +\left(z x-l^{2} y^{2}\right)\left[3 l^{2} y^{2}-\left(1+2 l^{3}\right) z x\right]^{2} \\
& +\left(x y-l^{2} z^{2}\right)\left[3 l^{2} z^{2}-\left(1+2 l^{3}\right) x y\right]^{2} \\
& +2\left(l^{2} y z-l x^{2}\right)\left[3 l^{2} y^{2}-\left(1+2 l^{3}\right) z x\right]\left[3 l^{2} z^{2}-\left(1+2 l^{3}\right) x y\right] \\
& +2\left(l^{2} z x-l y^{2}\right)\left[3 l^{2} z^{2}-\left(1+2 l^{3}\right) x y\right]\left[3 l^{2} x^{2}-\left(1+2 l^{3}\right) y z\right] \\
& +2\left(l^{2} x y-l z^{2}\right)\left[3 l^{2} x^{2}-\left(1+2 l^{3}\right) y z\right]\left[3 l^{2} y^{2}-\left(1+2 l^{3}\right) z x\right],
\end{aligned}
$$

the first three lines of which are

$$
\begin{aligned}
& 9 l^{4} x y z\left(x^{3}+y^{3}+z^{3}\right)-18 l^{2}\left(1+2 l^{3}\right) x^{2} y^{2} z^{2}+\left(1+2 l^{3}\right)^{2}\left(y^{3} z^{2}+z^{3} x^{3}+x^{3} y^{3}\right) \\
& -9 l^{6}\left(x^{6}+y^{6}+z^{6}\right)+6 l^{4}\left(1+2 l^{3}\right) x y z\left(x^{3}+y^{3}+z^{3}\right)-3 l^{2}\left(1+2 l^{3}\right)^{2} x^{2} y^{2} z^{2}
\end{aligned}
$$

or collecting and reducing,

$$
\begin{aligned}
& \left(-9 l^{6}\right. \\
& \text { ) }\left(x^{6}+y^{6}+z^{6}\right) \\
& +\left(1+4 l^{3}+4 l^{6} \quad\right)\left(y^{3} z^{3}+z^{3} x^{3}+x^{3} y^{3}\right) \\
& +\left(15 l^{4}+12 l^{7} \quad\right)\left(x^{3}+y^{3}+z^{3}\right) x y z \\
& +\left(-21 l^{2}-48 l^{5}-12 l^{8}\right) x^{2} y^{2} z^{2} \text {; }
\end{aligned}
$$


the second three lines are

$$
\begin{aligned}
& 18 l^{6}\left(y^{3} z^{3}+z^{3} x^{3}+x^{3} y^{3}\right)-12 l^{4}\left(1+2 l^{3}\right) x y z\left(x^{3}+y^{3}+z^{3}\right)+6 l^{2}\left(1+2 l^{3}\right)^{2} x^{2} y^{2} z^{2} \\
- & 54 l^{5} x^{2} y^{2} z^{2}+12 l^{3}\left(1+2 l^{3}\right)\left(y^{3} z^{3}+z^{3} x^{3}+x^{3} y^{3}\right)-2 l\left(1+2 l^{3}\right)^{2} x y z\left(x^{3}+y^{3}+z^{3}\right)
\end{aligned}
$$

or collecting and reducing,

$$
\begin{aligned}
& \left(12 l^{3}+42 l^{6}\right)\left(y^{3} z^{3}+z^{3} x^{3}+x^{3} y^{3}\right) \\
+ & \left(-2 l-20 l^{4}-32 l^{7}\right) \\
+ & \left(6 x^{3}+y^{3}+z^{3}\right) x y z \\
+ & \left(60 l^{5}+24 l^{8}\right) x^{2} y^{2} z^{2} .
\end{aligned}
$$

Hence in the first part replacing the top line by

$$
-9 l^{6}\left(x^{3}+y^{3}+z^{3}\right)^{2}+18 l^{6}\left(y^{3} z^{3}+z^{3} x^{3}+x^{3} y^{3}\right)
$$

and uniting the two parts, we find

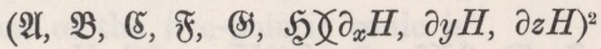

$$
\begin{aligned}
& =\quad\left(1+8 l^{3}\right)^{2}\left(y^{3} z^{3}+z^{3} x^{3}+x^{3} y^{3}\right) \\
& +\left(-9 l^{6}\right)\left(x^{3}+y^{3}+z^{3}\right)^{2} \\
& +\left(-2 l-5 l^{4}-20 l^{7}\right)\left(x^{3}+y^{3}+z^{3}\right) x y z \\
& +\left(-15 l^{2}-78 l^{5}+12 l^{8}\right) x^{2} y^{2} z^{2} \text {; }
\end{aligned}
$$

and referring to the Table No. 70, and writing $\Theta$ for $\Theta U$, we have

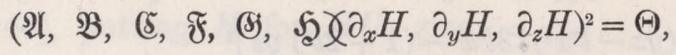

where $\Theta$ is the first of the three functions which may be chosen to represent the octicovariant of the cubic.

33. We have thus

$$
\Lambda=-\frac{4}{9} \frac{\Theta}{H^{3}}
$$

and thence

$$
D^{2} U-\left(\frac{2}{3} \frac{1}{H} D H-\frac{2}{27} \frac{\Theta}{H^{3}} D U\right) D U=0
$$

as the equation of the five-pointic conic: the investigation has been conducted by means of the canonical form of the equation of the cubic, but the form of the result shows that it applies to the equation of the cubic in any form whatever.

34. If, however, we continue to represent the cubic by the canonical equation

$$
x^{3}+y^{3}+z^{3}+6 l x y z=0,
$$

the result may be further reduced. We have, putting $U=0$, or writing $x^{3}+y^{3}+z^{3}=-6 l x y z$,

$$
H=-\left(1+8 l^{3}\right) x y z
$$


moreover, putting $U=0$, the Table No. 70 gives

$$
\Theta=\left(1+8 l^{3}\right)^{2}\left(y^{3} z^{3}+z^{3} x^{3}+x^{3} y^{3}\right)-3 l^{2} H^{2} ;
$$

or substituting for $H$ the last-mentioned value, and putting for shortness

we have

$$
Q=y^{3} z^{3}+z^{3} x^{3}+x^{3} y^{3}-3 l^{2} x^{2} y^{2} z^{2}
$$

$$
\Theta=\left(1+8 l^{3}\right)^{2} Q
$$

and with these values of $H$ and $Q$, the equation of the five-pointic conic is

where

$$
D^{2} U+\left(\frac{2}{3} \frac{1}{\left(1+8 l^{3}\right) x y z} D H-\frac{2}{27} \frac{Q}{\left(1+8 l^{3}\right) x^{3} y^{3} z^{3}} D U\right) D U=0,
$$

$$
\begin{aligned}
& D U=3\left\{\left(x^{2}+2 l y z\right) X+\left(y^{2}+2 l z x\right) Y+\left(z^{2}+2 l x y\right) Z\right\} \\
& D^{2} U=6\left\{\left(X^{2}+2 l Y Z\right) x+\left(Y^{2}+2 l Z X\right) y+\left(Z^{2}+2 l X Y\right) z\right\},
\end{aligned}
$$

or, as it will be convenient to write it,

$$
\begin{gathered}
=6(x, y, z, l x, l y, l z \gamma X, Y, Z)^{2}, \\
D H=\left(3 l^{2} x^{2}-\left(1+2 l^{3}\right) y z\right) X+\left(3 l^{2} y^{2}-\left(1+2 l^{3}\right) z x\right) Y+\left(3 l^{2} z^{2}-\left(1+2 l^{3}\right) x y\right) Z
\end{gathered}
$$

whence, finally, the equation of the five-pointic conic of the cubic

$$
X^{3}+Y^{3}+Z^{3}+6 l X Y Z=0
$$

at the point $(x, y, z)$ is

$$
\begin{aligned}
& 9\left(1+8 l^{3}\right) x^{3} y^{3} z^{3}(x, y, z, l x, l y, l z \gamma X, Y, Z)^{2} \\
& +\left\{\left(x^{2}+2 l y z\right) X+\left(y^{2}+2 l z x\right) \cdot Y+\left(z^{2}+2 l x y\right) Z\right\} \times
\end{aligned}
$$

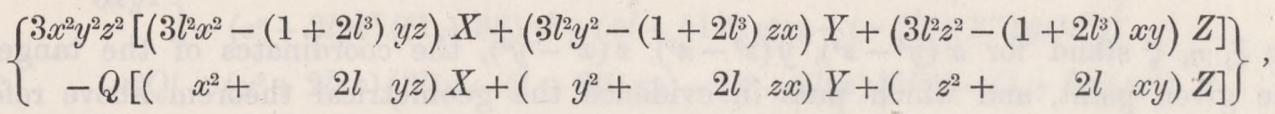

a result which I had previously obtained by a special method.

35. But the expression may be exhibited in a different form by a transformation suggested by a geometrical theorem of Mr Salmon's. In fact the tangent at the point $(x, y, z)$ meets the cubic in the tangential of this point, and the coordinates of the tangential are $x\left(y^{3}-z^{3}\right), y\left(z^{3}-x^{3}\right), z\left(x^{3}-y^{3}\right)$. Calling these $\xi, \eta$, $\zeta$, the equation of the tangent to the cubic at the tangential is

$$
\left(\xi^{2}+2 l \eta \zeta\right) X+\left(\eta^{2}+2 l \zeta \xi\right) Y+\left(\zeta^{2}+2 l \xi \eta\right) Z=0 .
$$

Now we have identically,

$$
\begin{aligned}
& 3 x^{2} y^{2} z^{2}\left\{\left(3 l^{2} x^{2}-\left(1+2 l^{3}\right) y z\right) X+\left(3 l^{2} y^{2}-\left(1+2 l^{3}\right) y z\right) Y+\left(3 l^{2} z^{2}-\left(1+2 l^{3}\right) z x\right) Z\right\} \\
& \quad-Q\left\{\left(x^{2}+2 l y z\right) X+\left(y^{2}+2 l z x\right) Y+\left(z^{2}+2 l x y\right) Z\right\} \\
& =\quad\left\{\left(\xi^{2}+2 l \eta \zeta\right) X+\left(\eta^{2}+2 l \zeta \xi\right) Y+\left(\zeta^{2}+2 l \xi \eta\right) Z\right\} \\
& +U\left\{x^{2}\left(-y^{3}-z^{2}+2 l y z\right) X+y^{2}\left(-z^{3}-x^{3}+2 l z x\right) Y+z^{2}\left(-x^{3}-y^{3}+2 l x y\right) Z\right\} .
\end{aligned}
$$

C. IV. 
In fact this relation will be true if only

$$
3 x^{2} y^{2} z^{2}\left(3 l^{2} x^{2}-\left(1+2 l^{3}\right) y z\right)-Q\left(x^{2}+2 l y z\right)-\left(\xi^{2}+2 l \eta \zeta\right)=U x^{2}\left(-y^{3}-z^{3}+2 l y z\right) ;
$$

and substituting for $\xi, \eta, \zeta$ and $Q$ their values, the left-hand side is

$$
\begin{aligned}
& 3 x^{2} y^{2} z^{2}\left(3 l^{2} x^{2}-\left(1+2 l^{3}\right) y z\right) \\
- & \left(x^{2}+2 l y z\right)\left(y^{3} z^{3}+z^{3} x^{3}+x^{3} y^{3}-3 l^{2} x^{2} y^{2} z^{2}\right) \\
- & x^{2}\left(y^{3}-z^{3}\right)^{2} \\
- & 2 l y z\left(z^{3}-x^{3}\right)\left(x^{3}-y^{3}\right)
\end{aligned}
$$

and expanding and reducing, the result is

$$
x^{2}\left(-\left(y^{3}+z^{3}\right)^{2}-x^{3}\left(y^{3}+z^{3}\right)\right)+2 l y z\left(-2 x^{3}\left(y^{3}+z^{3}\right)+x^{6}\right)+12 l^{2} x^{4} y^{2} z^{2}
$$

whence, dividing by $x^{2}$, the equation becomes

$$
\begin{aligned}
-\left(y^{3}+z^{3}\right)\left(x^{3}+y^{3}+z^{3}\right)+2 l x y z & \left(x^{3}-2\left(y^{3}+z^{3}\right)\right)+12 l^{2} x^{2} y^{2} z^{2} \\
& =\left(-y^{3}-z^{3}+2 l y z\right)\left(x^{3}+y^{3}+z^{3}+6 l x y z\right),
\end{aligned}
$$

which is identically true.

36. Hence in the identical equation putting $U=0$, we see that the equation of the five-pointic conic may be written

$$
\begin{aligned}
& 9\left(1+8 l^{3}\right) x^{3} y^{3} z^{3}(x, y, z, l x, l y, l z \gamma X, Y, Z)^{2} \\
+ & \left\{\left(x^{2}+2 l y z\right) X+\left(y^{2}+2 l z x\right) Y+\left(z^{2}+2 l x y\right) Z\right\} \times \\
& \left\{\left(\xi^{2}+2 l \eta \zeta\right) X+\left(\eta^{2}+2 l \zeta \xi\right) Y+\left(\zeta^{2}+2 l \xi \eta\right) Z\right\} \\
= & 0,
\end{aligned}
$$

where $\xi, \eta, \zeta$ stand for $x\left(y^{3}-z^{3}\right), y\left(z^{3}-x^{3}\right), z\left(x^{3}-y^{3}\right)$, the coordinates of the tangential of the given point, and which puts in evidence the geometrical theorem above referred to, viz.

THEOREM.-The common chord of the five-pointic conic and the polar conic is the tangent to the cubic at the tangential of the given point.

37. The five-pointic conic meets the cubic in the point of contact, considered as five coincident points, and in a remaining sixth point or point of simple intersection. The process by which I originally obtained the equation of the five-pointic conic, led also to the equation of the line joining the point of contact with the point of simple intersection: the equation of this line is

$$
\begin{aligned}
& 9 x^{3} y^{3} z^{3}\left\{\begin{array}{r}
X x\left(\left(1+8 l^{3}\right) x^{4}+\left(4 l+41 l^{4}\right) x^{2} y z+\left(-2 l^{2}+2 l^{5}\right) y^{2} z^{2}\right) \\
+Y y\left(\left(1+8 l^{3}\right) y^{4}+\left(4 l+41 l^{4}\right) y^{2} z x+\left(-2 l^{2}+2 l^{5}\right) z^{2} x^{2}\right) \\
+Z z\left(\left(1+8 l^{3}\right) z^{4}+\left(4 l+41 l^{4}\right) z^{2} x y+\left(-2 l^{2}+2 l^{5}\right) x^{2} y^{2}\right)
\end{array}\right\} \\
&-6 Q x^{2} y^{2} z^{2}\left\{X\left(3 l^{2} x^{2}-\left(1+2 l^{3}\right) y z\right)+Y\left(3 l^{2} y^{2}-\left(1+2 l^{3}\right) z x\right)+Z\left(3 l^{2} z^{2}-\left(1+2 l^{3}\right) x y\right)\right\} \\
&+Q^{2} \quad\left\{X\left(x^{2}+2 l y z\right)+Y\left(y^{2}+2 l z x\right)+Z\left(z^{2}+2 l x y\right)\right\}=0 .
\end{aligned}
$$


38. If the conic meet the cubic in six coincident points, that is, if the point of contact be a singular point of the kind already spoken of, or, as we may term it, a sextactic point, then the last-mentioned line must coincide with the tangent at the point. Represent for a moment the equation of the line by

$$
A X+B Y+C Z=0,
$$

then this line is to coincide with the line

or we must have

$$
\left(x^{2}+2 l y z\right) X+\left(y^{2}+2 l z x\right) Y+\left(z^{2}+2 l x y\right) Z=0,
$$

$$
\begin{aligned}
& B\left(z^{2}+2 l x y\right)-C\left(y^{2}+2 l z x\right)=0 \\
& C\left(x^{2}+2 l y z\right)-A\left(z^{2}+2 l x y\right)=0 \\
& A\left(y^{2}+2 l z x\right)-B\left(x^{2}+2 l y z\right)=0
\end{aligned}
$$

which must be equivalent to a single condition. The terms $A, B, C$, which contain

$$
x^{2}+2 l y z, \quad y^{2}+2 l z x, \quad z^{2}+2 l x y
$$

respectively, may, it is clear, be omitted, and omitting also a factor $3 x^{2} y^{2} z^{2}$, we may write

$$
\begin{aligned}
& A=3 x^{2} y z\left(\left(1+8 l^{3}\right) x^{4}+\left(4 l+41 l^{4}\right) x^{2} y z+\left(-2 l^{2}+2 l^{5}\right) y^{2} z^{2}\right)-2 Q\left(3 l^{2} x^{2}-\left(1+2 l^{3}\right) y z\right) \\
& B=3 x y^{2} z\left(\left(1+8 l^{3}\right) y^{4}+\left(4 l+41 l^{4}\right) y^{2} z x+\left(-2 l^{2}+2 l^{5}\right) z^{2} x^{2}\right)-2 Q\left(3 l^{2} y^{2}-\left(1+2 l^{3}\right) z x\right),
\end{aligned}
$$

and the like value for $C$. The last of the three equations is

$$
\begin{aligned}
& 3 x y z\left\{\begin{array}{r}
\left(y^{2}+2 l z x\right)\left(\left(1+8 l^{3}\right) x^{5}+\left(4 l+41 l^{4}\right) x^{3} y z+\left(-2 l^{2}+2 l^{5}\right) x y^{2} z^{2}\right) \\
-\left(x^{2}+2 l y z\right)\left(\left(1+8 l^{3}\right) y^{5}+\left(4 l+41 l^{4}\right) y^{3} z x+\left(-2 l^{2}+2 l^{5}\right) y z^{2} x^{2}\right)
\end{array}\right\} \\
& -2 Q\left\{\quad\left(y^{2}+2 l z x\right)\left(3 l^{2} x^{2}-\left(1+2 l^{3}\right) y z\right)-\left(x^{2}+2 l y z\right)\left(3 l^{2} y^{2}-\left(1+2 l^{3}\right) z x\right)\right\} \\
& =0,
\end{aligned}
$$

where the function on the left hand is

$$
\left.\begin{array}{c}
=3 x y z\left\{\begin{array}{c}
\left(1+8 l^{3}\right)\left(x^{5} y^{2}-x^{2} y^{5}+2 l\left(x^{6} z-y^{6} z\right)\right) \\
+\left(4 l+41 l^{4}\right) 2 l\left(x^{4} y z^{2}-x y^{4} z^{2}\right) \\
+\left(-2 l^{2}+2 l^{5}\right)\left(x y^{4} z^{2}-x^{4} y z^{2}\right)
\end{array}\right\} \\
-2 Q\left\{\left(1+2 l^{3}\right)\left(y^{3} z-x^{3} z\right)+6 l^{2}\left(x^{3} z-y^{3} z\right)\right\}
\end{array}\right\}
$$

or, what is the same thing, throwing out the factor $z$, it is

$$
\begin{aligned}
=\left\{3\left(1+8 l^{3}\right) x^{3} y^{3}+6 l\left(1+8 l^{3}\right) x y z\left(x^{3}+y^{3}\right)+30 l^{2}\left(1+8 l^{3}\right) x^{2} y^{2} z^{2}\right\}\left(x^{3}-y^{3}\right) \\
-2 Q\left(1+8 l^{3}\right)\left(x^{3}-y^{2}\right) ;
\end{aligned}
$$

or throwing out the factor $\left(1+8 l^{3}\right)$ and substituting for $Q$ its value, it is

$$
=\left\{3 x^{3} y^{3}+6 l x y z\left(x^{3}+y^{3}\right)+30 l^{2} x^{2} y^{2} z^{2}-2\left(y^{3} z^{3}+z^{3} x^{3}+x^{3} y^{3}-3 l^{2} x^{2} y^{2} z^{2}\right)\right\}\left(x^{3}-y^{3}\right) .
$$


The first factor, reducing by the equation $x^{3}+y^{3}+z^{3}+6 l x y z=0$, is

$$
\begin{aligned}
& =3 x^{3} y^{3}-\left(x^{3}+y^{3}\right)\left(x^{3}+y^{3}+z^{3}\right)+\left(x^{3}+y^{3}+z^{3}\right)^{2}-2\left(y^{3} z^{3}+z^{3} x^{3}+x^{3} y^{3}\right) \\
& =3 x^{3} y^{3}+z^{3}\left(x^{3}+y^{3}+z^{3}\right)-2\left(y^{3} z^{3}+z^{3} x^{3}+x^{3} y^{3}\right) \\
& =z^{6}-z^{3}\left(x^{3}+y^{3}\right)+x^{3} y^{3} \\
& =\left(z^{3}-x^{3}\right)\left(z^{3}-y^{3}\right)
\end{aligned}
$$

39. Hence putting for the moment

$$
M=\left(y^{3}-z^{3}\right)\left(z^{3}-x^{3}\right)\left(x^{3}-y^{3}\right),
$$

it appears that the last of the three equations is $M z=0$; the first and second are of course $M x=0$ and $M y=0$, and the required condition is $M=0$, that is,

$$
\left(y^{3}-z^{3}\right)\left(z^{3}-x^{3}\right)\left(x^{3}-y^{3}\right)=0,
$$

the equation which, combined with the equation of the curve

$$
x^{3}+y^{3}+z^{3}+6 l x y z=0,
$$

gives the sextactic points. There are consequently twenty-seven such points, and it is at once seen that these are the points of contact of the tangents to the cubic from the points of inflexion, or, what is the same thing, that the twenty-seven sextactic points form nine groups of three each, such that the three points of a group have for their common tangential one of the nine points of inflexion. In fact, let $\omega$ be a cube root (real or imaginary) of unity, the three sextactic points of one of the groups will be given by

$$
\left\{\begin{aligned}
x-\omega y & =0 \\
x^{3}+y^{3}+z^{3}+6 l x y z & =0
\end{aligned}\right.
$$

Now consider the tangential of any one of these points, its coordinates are

$$
\begin{aligned}
& x_{1}=x\left(y^{3}-z^{3}\right), \\
& y_{1}=y\left(z^{3}-x^{3}\right), \\
& z_{1}=z\left(x^{3}-y^{3}\right)
\end{aligned}
$$

or, reducing by the equation $x-\omega y=0 ; x_{1}=\omega y\left(x^{3}-z^{3}\right), y_{1}=-y\left(x^{3}-z^{3}\right), z_{1}=0$, or, what is the same thing, $x_{1}+\omega y_{1}=0, z_{1}=0$; that is, the point $\left(x_{1}, y_{1}, z_{1}\right)$ is one of the points of inflexion. This is the construction of the sextactic points obtained by Pliicker and Steiner.

40. Reverting to the equation of the line joining the point of contact of the fivepointic conic with the point of simple intersection, this meets the cubic in a third point, and $\mathrm{Mr}$ Salmon has shown that this third point is in fact the second tangential (tangential of the tangential) of the point of contact, or, what is the same thing:

Theorem. The point of simple intersection of the cubic and the five-pointic conic is the third point of intersection with the cubic, of the line joining the point of contact with the second tangential of this point. 
41. I have not sought to verify this theorem by my formulæ. I remark, that combining it with the before-mentioned theorem, the five-pointic conic is completely determined as follows; viz.

Theorem. The five-pointic conic touches the conic at the point of contact (two conditions); it passes through the two points in which the polar conic is intersected by the tangent to the cubic at the tangential of the point of contact (two conditions); and it passes through the point which is the third point of intersection with the cubic of the line joining the point of contact with its second tangential.

42. The construction for the point of simple intersection leads at once to that for the sextactic points; in fact, consider a point having for its tangential a point of inflexion: a point of inflexion is its own tangential, and the second tangential of the first-mentioned point is therefore the point of inflexion: the line joining the point with the second tangent is therefore the tangent at the point, and the point of simple intersection coincides with the point itself, that is, the point in question is a sextactic point.

43. I represent the equation of the five-pointic conic by

the value of $a$ is

$$
(a, b, c, f, g, h \gamma X, Y, Z)^{2}=0 ;
$$

$$
\begin{aligned}
= & 9\left(1+8 l^{3}\right) x^{4} y^{3} z^{3} \\
& +3 x^{2} y^{2} z^{2}\left(3 l^{2} x^{2}-\left(1+2 l^{3}\right) y z\right)\left(x^{2}+2 l y z\right) \\
& -Q\left(x^{2}+2 l y z\right)^{2},
\end{aligned}
$$

in which equation

$$
Q=y^{3} z^{3}+z^{3} x^{3}+x^{3} y^{3}-3 l^{2} x^{2} y^{2} z^{2}
$$

or, reducing by the equation $x^{3}+y^{3}+z^{3}+6 l x y z=0$,

that is,

$$
Q=y^{3} z^{3}+x^{3}\left(-x^{3}-6 l x y z\right)-3 l^{2} x^{2} y^{2} z^{2},
$$

and we have

$$
-Q=x^{6}+6 l x^{4} y z+3 l^{2} x^{2} y^{2} z^{2}-y^{3} z^{3},
$$

$$
\begin{aligned}
a= & 9\left(1+8 l^{3}\right) x^{4} y^{3} z^{3} \\
& +3 x^{2} y^{2} z^{2}\left(3 l^{2} x^{4}+\left(-1+4 l^{3}\right) x^{2} y z-2 l\left(1+2 l^{3}\right) y^{2} z^{2}\right) \\
& +\left(x^{6}+6 l x^{4} y z+3 l^{2} x^{2} y^{2} z^{2}-y^{3} z^{3}\right)\left(x^{4}+4 x l^{2} y z+4 l^{2} y^{2} z^{2}\right) .
\end{aligned}
$$

We have in like manner

$$
\begin{aligned}
2 f= & 18\left(1+8 l^{3}\right) l x^{4} y^{3} z^{3} \\
& +3 x^{2} y^{2} z^{2}\left\{\left(3 l^{2} y^{2}-\left(1+2 l^{3}\right) z x\right)\left(z^{2}+2 l x y\right)+\left(3 l^{2} z^{2}-\left(1+2 l^{3}\right) x y\right)\left(y^{2}+2 l z x\right)\right\} \\
& -2 Q\left(y^{2}+2 l z x\right)\left(z^{2}+2 l x y\right)
\end{aligned}
$$

the coefficient of $3 x^{2} y^{2} z^{2}$ in the second line is

$$
=6 l^{2} y^{2} z^{2}+\left(-1+4 l^{3}\right) x\left(y^{3}+z^{3}\right)-4 l\left(1+2 l^{3}\right) x^{2} y z ;
$$

or reducing by the equation of the curve,

$$
=\left(1-4 l^{3}\right) x^{4}+\left(2 l-32 l^{4}\right) x^{2} y z+6 l^{2} y^{2} z^{2} ;
$$


and the coefficient of $-2 Q$ is

$$
=y^{2} z^{2}+2 l x\left(y^{3}+z^{3}\right)+4 l^{2} x^{2} y z
$$

or reducing by the equation of the curve,

We thus have

$$
=-2 l x^{4}-8 l^{2} x^{2} y z+y^{2} z^{2}
$$

$$
\begin{aligned}
2 f= & 18 l\left(1+8 l^{3}\right) x^{4} y^{3} z^{3} \\
& +3 x^{2} y^{2} z^{2}\left(\left(1-4 l^{3}\right) x^{4}+\left(2 l-32 l^{4}\right) x^{2} y z+6 l^{2} y^{2} z^{2}\right) \\
& +2\left(x^{6}+6 l x^{4} y z+3 l^{2} x^{2} y^{2} z^{2}-y^{3} z^{3}\right)\left(-2 l x^{4}-8 l^{2} x^{2} y z+y^{2} z^{2}\right) .
\end{aligned}
$$

Reducing the expressions of $a$ and $2 f$, we find for the coefficients $(a, b, c, f, g, h$ ),

$$
\left\{\begin{array}{l}
a=\quad x^{10}+10 l x^{8} y z+40 l^{2} x^{6} y^{2} z^{2}+\left(5+120 l^{3}\right) x^{4} y^{3} z^{3}-10 l x^{2} y^{4} z^{4}-4 l^{2} y^{5} z^{5} \\
\vdots \\
2 f=-4 l x^{10}-40 l^{2} x^{8} y z+\left(5-120 l^{3}\right) x^{6} y^{2} z^{2}+40 l x^{4} y^{3} z^{3}+8 l^{2} x^{2} y^{4} z^{4}-2 y^{5} z^{5},
\end{array}\right.
$$

which gives the completely developed form of the equation of the five-pointic conic.

44. I investigate the coordinates of the point of simple intersection of the cubic and the five-pointic conic as follows: the equations of the two curves are

or if we write

$$
\begin{aligned}
& X^{3}+Y^{3}+Z^{3}+6 l X Y Z=0 \\
& (a, b, c, f, g, h \gamma X, Y, Z)^{2}=0 ;
\end{aligned}
$$

$$
\begin{array}{ll}
\alpha=1, & A=c, \\
\gamma=6 l X Y, & B=2(g X+f Y), \\
\delta=X^{3}+Y^{3}, & C=a X^{2}+2 h X Y+b Y^{2},
\end{array}
$$

then the two equations are

$$
\begin{aligned}
& \alpha Z^{3}+\gamma Z+\delta=0, \\
& A Z^{2}+B Z+C=0,
\end{aligned}
$$

and the result of the elimination of $Z$ will be

$$
\left(\alpha Z_{1}^{3}+\gamma Z_{1}+\delta\right)\left(\alpha Z_{2}^{3}+\gamma Z_{2}+\delta\right)=0,
$$

where $Z_{1}, Z_{2}$ are the roots of the equation $A Z^{2}+B Z+C=0$; that is, we have

$$
\begin{aligned}
& \alpha^{2} \quad C^{3} \\
& + \text { ay } C\left(B^{2}-2 A C\right) \\
& +\alpha \delta\left(-B^{3}+2 A B C\right) \\
& +\gamma^{2} C A^{2} \\
& -\gamma \delta B A^{2} \\
& +\delta^{2} A^{3} \\
& =0 \text { ； }
\end{aligned}
$$


and substituting for $A, B, C$ their values, but attending only to the terms which involve $X^{6}$ and $Y^{6}$, the result is

$$
\left(a^{3}+c^{3}-8 g^{3}+6 a c g\right) X^{6}+\ldots+\left(b^{3}+c^{3}-8 f^{3}+6 b c f\right) Y^{6}=0 .
$$

45. But the result of the elimination must obviously be

$$
(X y-Y x)^{5}\left(X y_{1}-Y x_{1}\right)=0,
$$

if $\left(x_{1}, y_{1}, z_{1}\right)$ are the coordinates of the point of simple intersection. Comparing the two results, and forming the analogous third equation, we may write

$$
\begin{aligned}
& x^{5} x_{1}=b^{3}+c^{3}-8 f^{3}+6 b c f, \\
& y^{5} y_{1}=c^{3}+a^{3}-8 g^{3}+6 c a g, \\
& z^{5} z_{1}=a^{3}+b^{3}-8 h^{3}+6 a b h,
\end{aligned}
$$

where the value of $x^{5} x_{1}$ may also be written $(b+c-2 f)\left(b+c \omega-2 \omega^{2} f\right)\left(b+c \omega^{2}-2 \omega f\right)$, $\omega$ being an imaginary cube root of unity, and so for the other two terms. The factors of $x^{5} x_{1}$ might be calculated from the identical equation

$$
\begin{aligned}
b Y^{2}+2 f Y Z+c Z^{2}= & 9\left(1+8 l^{3}\right) x^{3} y^{3} z^{3}\left(y Y^{2}+z Z^{2}+2 l x Y Z\right) \\
+ & \left\{\left(y^{2}+2 l z x\right) Y+\left(z^{2}+2 l x y\right) Z\right\} \times \\
& \left\{\begin{array}{c}
3 x^{2} y^{2} z^{2}\left[\left(3 l^{2} y^{2}-\left(1+2 l^{3}\right) z x\right) Y+\left(3 l^{2} z^{2}-\left(1+2 l^{3}\right) x y\right) Z\right] \\
-Q\left[\left(y^{2}+2 l z x\right) Y+\left(z^{2}+2 l x y\right) Z\right]
\end{array}\right\} .
\end{aligned}
$$

I remark, that putting $x=0$, we have

$$
b Y^{2}+2 f Y Z+c Z^{2}=-y^{3} z^{3}\left(y^{2} Y+z^{2} Z\right)^{2},
$$

and hence writing 1 for $Y$, and $-1,-\omega,-\omega^{2}$ for $Z$, we have

$$
b+c-2 f=-y^{3} z^{3}\left(y^{2}-z^{2}\right)^{2}, b+c \omega-2 \omega^{2} f=-y^{3} z^{3}\left(y^{2}-\omega^{4} z^{2}\right)^{2}, b+c \omega^{2}-2 \omega f=-y^{3} z^{3}\left(y^{2}-\omega^{2} z^{2}\right)^{2} ;
$$

and hence the product of the three factors is $-y^{9} z^{9}\left(y^{2}-z^{2}\right)^{2}\left(y^{2}-\omega^{2} z^{2}\right)^{2}\left(y^{2}-\omega^{4} z^{2}\right)^{2}$, which is equal to $-y^{9} z^{9}\left(y^{3}-z^{3}\right)^{2}\left(y^{3}+z^{3}\right)^{2}$, which vanishes in virtue of the assumed question $x=0$. This shows that the function $b^{3}+c^{3}-8 f^{3}+6 b c f$ contains the factor $x$. I have not verified a posteriori, but I assume it to be true, that it contains in fact the factor $x^{6}$, and consequently that the expressions for $x_{1}, y_{1}, z_{1}$ are rational and integral functions of $(x, y, z)$ of the degree 25, and containing respectively the factors $x, y, z$.

46. In the theory of the cubic, a point which depends linearly upon a given point may be termed a derivative of such point. According to a very beautiful theorem of Professor Sylvester's, the coordinates of a derivative point are necessarily rational and integral functions of a square degree of the coordinates $(x, y, z)$ of the given point; and moreover, there is but one derivative point having its coordinates of any given square degree $m^{2}$, or, as we may express it, only one derivative point of the degree $m^{2}$. The successive tangentials are derivative points of the degrees $4,16,64, \& c$; t the third point of intersection with the cubic, of the line joining two 
derivative points of the degrees $m^{2}$ and $n^{2}$ respectively, is a derivative point of the degree $(m \pm n)^{2}$. Thus the third point of intersection with the cubic, of the line joining the given point with its second tangential, is a derivative point of the degree $(4 \pm 1)^{2}$, and it is easy to see that the degree is not 9 ; it is therefore 25 . The point of simple intersection of the five-pointic conic is a derivative point of the degree 25; it is therefore, according to Professor Sylvester's general theory, identical with the point given by the former construction; this agrees with the beforementioned theorem of Mr Salmon.

\section{Independent investigation for the Cubic.}

47. The following is, in substance, the method by which I first obtained the equation of the five-pointic conic, for the cubic

$$
X^{3}+Y^{3}+Z^{3}+6 l X Y Z=0 .
$$

Write for shortness

$$
\begin{aligned}
& U=x^{3}+y^{3}+z^{3}+6 l x y z, \\
& V=\left(x^{2}+2 l y z\right) X+\left(y^{2}+2 l z x\right) Y+\left(z^{2}+2 l x y\right) Z, \\
& W=\left(X^{2}+2 l Y Z\right) x+\left(Y^{2}+2 l X Z\right) y+\left(Z^{2}+2 l X Y\right) z, \\
& \Upsilon=X^{3}+Y^{3}+Z^{3}+6 l X Y Z, \\
& \mathbf{P}=a x+b y+c z, \\
& \Pi=a X+b Y+c Z ;
\end{aligned}
$$

then, $X, Y, Z$ being current coordinates, and $x, y, z$ the coordinates of a point of the cubic (so that $U=0$ ), the equation of the cubic will be

$$
\Upsilon=0,
$$

and the equation of a conic having with it an ordinary (two-pointic) contact at the point $(x, y, z)$, will be $\left.{ }^{1}\right)$

$$
2 W-\Pi V=0 .
$$

48. Now imagine from the point of contact lines drawn to the other four intersections of the two curves; in the case of the five-pointic conic, three of these lines will coincide with the tangent $V=0$, and the remaining line will be the line joining the point of contact with the point of simple intersection. The equations of the lines in question can be found by Joachimsthal's theorem, viz. if $(x, y, z)$ be the coordinates of a given point, and $(X, Y, Z)$ current coordinates, then if in the equations of any two curves we substitute for the coordinates, $\lambda x+\mu X, \lambda y+\mu Y, \lambda z+\mu Z$, and between the equations so obtained eliminate $\lambda, \mu$, the resulting equation will be that

1 I have introduced the factor 2, to make this correspond with the form $D^{2} U-\Pi D U=0$, in the case in question, $m=3$. 
of the lines drawn from the point $(x, y, z)$ to the points of intersection of the two curves. The point $(x, y, z)$ is any point whatever, and it may therefore be a point of intersection, or, as in the present instance, a point of contact of the two curves; the only difference is, that in either case the degree of each equation as regards $(\lambda, \mu)$ is reduced by unity, and the degree of the resulting equation in $X, Y, Z$ is also reduced by unity: in the case of a point of simple intersection this is the only reduction; but in the case of a point of contact, the resulting equation contains the equation of the tangent as a factor, and rejecting this factor, the reduction in degree is two units.

49. Applying the method to the two equations, $\Upsilon=0,2 W-\Pi V=0$, and substituting therein for the original current coordinates $X, Y, Z$ the values $\lambda x+\mu X$, $\lambda y+\mu Y, \lambda z+\mu Z$, the equations become

$$
\begin{aligned}
& \lambda^{3} U+3 \lambda^{2} \mu V+3 \lambda \mu^{2} W+\mu^{3} \Upsilon=0, \\
& 2\left(\lambda^{2} U+2 \lambda \mu V+\mu^{2} W\right)-(\lambda P+\mu \Pi)(\lambda U+\mu V)=0 ;
\end{aligned}
$$

or writing $U=0$, and omitting from each equation the factor $\mu$, the equations become

$$
\begin{aligned}
& \lambda^{2} .3 V+\lambda \mu .3 W+\mu^{2} \Upsilon=0, \\
& \lambda(4-P) V+\mu(2 W-\Pi V)=0 ;
\end{aligned}
$$

and putting in the first equation $\lambda=2 W-\Pi V, \mu=-(4-P) V$, the result of the elimination contains the factor $V$, rejecting which it becomes

$$
3(2 W-\Pi V)^{2}-3(4-P)(2 W-\Pi V) W+(4-P)^{2} V \Upsilon=0,
$$

which is of the fourth degree in $(X, Y, Z)$, as it should be, and represents therefore the lines drawn from the point of contact to the other four points of intersection of the conic and cubic.

50. The equation may be written

$$
-3(2 W-\Pi V)((2-P) W+\Pi V)+(4-P)^{2} V \Upsilon=0,
$$

and we obtain at once the condition that this may contain the factor $V$, viz. this condition is

$$
P=2 ;
$$

and if this be satisfied the conic will have a three-pointic conic, and there will be three other points of intersection. And writing $P=2$, and throwing out the factor $V$, we find

$$
3 \Pi^{2} V-6 \Pi W+4 \Upsilon=0
$$

for the equation of the lines from the point of contact to the three points of intersection. And we have now to determine $\Pi$ so that the function on the left hand may divide by $V^{2}$.

c. IV. 
51. I simplify my original method by the use of a theorem of Mr Salmon's, viz. writing

$$
\begin{aligned}
& \Upsilon=X^{3}+Y^{3}+Z^{3}+6 l X Y Z, \quad \mid \mathcal{G}=l^{2}\left(X^{3}+Y^{3}+Z^{3}\right)-\left(1+2 l^{3}\right) X Y Z, \\
& W=\left(X^{2}+2 l Y Z\right) x+\ldots \quad, \quad \mathfrak{S}_{1}=\left(3 l^{2} X^{2}-\left(1+2 l^{3}\right) Y Z \quad\right) x+\ldots \text {, } \\
& V=\left(x^{2}+2 l y z \quad\right) X+\ldots \quad, \quad H_{1}=\left(3 l^{2} x^{2}-\left(1+2 l^{3}\right) y z \quad\right) X+\cdots, \\
& U=x^{3}+y^{3}+z^{3}+6 l x y z \quad, \quad H=l^{2}\left(x^{3}+y^{3}+z^{3}\right)-\left(1+2 l^{3}\right) x y z \quad \text {, }
\end{aligned}
$$

we have identically

$$
\Upsilon H-U \mathfrak{H}_{\mathfrak{c}}=W H_{1}-V \mathfrak{H}_{1}
$$

and in the present case, since $U=0$,

$$
\Upsilon H=W H_{1}-V \mathfrak{S}_{1} .
$$

Hence, multiplying by $H$ and substituting this value of $\Upsilon H$, the equation becomes

$$
3 H \Pi^{2} V-6 H \Pi W+4 W H_{1}-4 V \mathfrak{g}_{1}=0,
$$

or, as we may write it,

$$
V\left(3 H \Pi^{2}-4 \mathfrak{J}_{1}\right)+2 W\left(2 H_{1}-3 H \Pi\right)=0
$$

and we can at once make the equation divide by $V$, viz. by assuming

$$
\Pi=\frac{2}{3} \frac{H_{1}}{H}+\frac{1}{2} \Lambda V
$$

where $\Lambda$ is arbitrary: we have thus a four-pointic contact. And substituting for $\Pi$, and throwing out the factor $V$, the equation becomes

$$
3 H\left(\frac{2}{3} \frac{H_{1}}{H}+\frac{1}{2} \Lambda V\right)^{2}-4 \mathfrak{H}_{1}+2 W\left(\frac{3}{2} H \Lambda\right)=0
$$

or reducing,

$$
16\left(H_{1}^{2}-3 H \mathfrak{g}_{1}\right)-36 H^{2} \Lambda W+24 H H_{1} \Lambda V+9 H^{2} \Lambda^{2} V^{2}=0
$$

which is the equation of the lines drawn from the point of contact to the remaining two points of intersection.

52. I write for greater convenience

$$
\Lambda=-\frac{4}{9} \frac{\Theta}{H^{3}}
$$

$\Theta$ being as yet indeterminate; the equation is thus reduced to

$$
9 H^{3}\left\{H\left(H_{1}^{2}-3 H \mathfrak{S}_{1}\right)+\Theta W\right\}-6 H^{2} H_{1} \Theta V+\Theta^{2} V^{2}=0,
$$

and we have then to determine $\Theta$ so that the left-hand side may divide by $V$; or, what is the same thing, we must determine $\Theta$ so that

$$
H\left(H_{1}^{2}-3 H \mathfrak{H}_{1}\right)+\Theta W
$$


may divide by $V$. This implies the existence of an identical equation,

$$
H\left(H_{1}{ }^{2}-3 H \mathfrak{S}_{1}\right)+\Theta W=M U+N V,
$$

which for $U=0$ would give the decomposition in question; but I have not investigated the values of $M$ and $N$. I assume at the outset $U=0$, and putting as before

and writing also

$$
Q=y^{3} z^{3}+z^{3} x^{3}+x^{3} y^{3}-3 l^{2} x^{2} y^{2} z^{2}
$$

$$
\begin{aligned}
S= & X x\left[\left(1+8 l^{3}\right) x^{4}+\left(4 l+41 l^{4}\right) x^{2} y z+\left(-2 l^{2}+2 l^{5}\right) y^{2} z^{2}\right] \\
& +Y y\left[\left(1+8 l^{3}\right) y^{4}+\left(4 l+41 l^{4}\right) y^{2} z x+\left(-2 l^{2}+2 l^{5}\right) z^{2} x^{2}\right] \\
& +Z z\left[\left(1+8 l^{3}\right) z^{4}+\left(4 l+41 l^{4}\right) z^{2} x y+\left(-2 l^{2}+2 l^{5}\right) x^{2} y^{2}\right],
\end{aligned}
$$

I remark that for $U=0$ we have

$$
-x y z H_{1}{ }^{2}+V S+\left(1+8 l^{3}\right)\left(Q W-3 x^{2} y^{2} z^{2} \mathfrak{S}_{1}\right)=0,
$$

an equation which, observing that

and assuming also

$$
H=-\left(1+8 l^{3}\right) x y z
$$

may be written

$$
\Theta=\left(1+8 l^{3}\right)^{2} Q,
$$

$$
H\left(H_{1}{ }^{2}-3 H \mathfrak{S}_{1}\right)+\Theta W=-\left(1+8 l^{3}\right) V S,
$$

which gives the required decomposition, viz., $\Theta$ having this value, the conic will have a five-pointic contact. Reducing by the last equation, and throwing out the factor $V$, we find

$$
-9 H^{3}\left(1+8 l^{3}\right) V-6 H^{2} \Theta H_{1}+\Theta^{2} V=0
$$

for the equation of the line joining the point of contact with the point of simple intersection. And 'if in this equation we write $H=-\left(1+8 l^{3}\right) x y z$, and $\Theta=\left(1+8 l^{3}\right)^{2} Q$, we obtain, finally, for the equation of the line in question,

$$
9 x^{3} y^{3} z^{3} S-6 x^{2} y^{2} z^{2} Q H_{1}+Q^{2} V=0,
$$

which is the before-mentioned result.

53. It only remains to verify the assumed equation

We may write

$$
-x y z H_{1}{ }^{2}+V S+\left(1+8 l^{3}\right)\left(Q W-3 x^{2} y^{2} z^{2} \mathfrak{S}_{1}\right)=0 .
$$

and then observing that

$$
-Q=x^{6}+6 l x^{4} y z+3 l^{2} x^{2} y^{2} z^{2}-y^{3} z^{3},
$$

$$
\begin{aligned}
& W=x X^{2}+\ldots+\quad 2 l x Y Z+\ldots \\
& \mathfrak{S}_{1}=3 l^{2} x X^{2}+. .-\left(1+2 l^{3}\right) x Y Z-\ldots
\end{aligned}
$$


we find at once

$$
-\left(1+8 l^{3}\right)\left\{\begin{array}{c}
\left(1+8 l^{3}\right)\left(Q W-3 x^{2} y^{2} z^{2} \mathfrak{F}_{1}\right)= \\
\left(x^{7}+6 l x^{5} y z+12 l^{2} x^{3} y z-x y^{3} z^{3}\right) X^{2} \\
\vdots \\
+\left(2 l x^{7}+12 l^{2} x^{5} y z-3 x^{3} y^{2} z^{2}-2 l x y^{3} z^{3}\right) Y Z \\
\vdots
\end{array}\right.
$$

Next writing

$$
\begin{aligned}
& H_{1}=\left(3 l^{2} x^{2}-\left(1+2 l^{3}\right) y z\right) X+\ldots \\
& V=\left(x^{2}+2 l y z\right) X+\ldots \\
& S=x\left\{\left(1+8 l^{3}\right) x^{4}+\left(4 l+41 l^{4}\right) x^{2} y z+\left(-2 l^{2}+2 l^{5}\right) y^{2} z^{2}\right\} X+\ldots
\end{aligned}
$$

and forming the expression for

$$
-x y z H_{1}^{2}+V S
$$

the coefficient of $X^{2}$ is

$$
\begin{array}{ll}
-x y z & \left\{3 l^{2} x^{2}-\left(1+2 l^{3}\right) y z\right\}^{2} \\
+\left(x^{2}+2 l y z\right) x & \left\{\left(1+8 l^{3}\right) x^{4}+\left(4 l+41 l^{4}\right) x^{2} y z+\left(-2 l^{2}+2 l^{5}\right) y^{2} z^{2}\right\},
\end{array}
$$

which is

$$
=\left(1+8 l^{3}\right)\left(x^{7}+6 l x^{5} y z+12 l^{2} x^{3} y^{2} z^{2}-x y^{3} z^{5}\right) ;
$$

the coefficient of $Y Z$ is

which is

$$
\begin{aligned}
& -2 x y z\left(3 l^{2} y^{2}-\left(1+2 l^{3}\right) z x\right)\left(3 l^{2} z^{2}-\left(1+2 l^{3}\right) x y\right) \\
& +\left(y^{2}+2 l z x\right) z\left\{\left(1+8 l^{3}\right) z^{4}+\left(4 l+41 l^{4}\right) z^{2} x y+\left(-2 l^{2}+2 l^{5}\right) x^{2} y^{2}\right\} \\
& +\left(z^{2}+2 l x y\right) y\left\{\left(1+8 l^{3}\right) y^{4}+\left(4 l+41 l^{4}\right) y^{2} z x+\left(-2 l^{2}+2 l^{5}\right) x^{2} y^{2}\right\}
\end{aligned}
$$

$$
=\left(1+8 l^{3}\right)\left\{y^{2} z^{2}\left(y^{3}+z^{3}\right)+8 l x y^{3} z^{3}+12 l^{2} x^{2} y z\left(y^{3}+z^{3}\right)+2 l x\left(y^{6}+z^{6}\right)-2 x^{3} y^{2} z^{2}\right\}
$$

or substituting for $y^{3}+z^{3}$ and $y^{6}+z^{6}$ the values $-x^{3}-6 l x y z$ and $\left(x^{3}+6 l x y z\right)^{2}-2 y^{3} z^{3}$ respectively, this is

$$
=\left(1+8 l^{3}\right)\left(2 l x^{7}+12 l^{2} x^{5} y z-3 x^{3} y^{2} z^{2}-2 l x y^{3} z^{3}\right) .
$$

We have thus

$$
\begin{aligned}
& -x y z H_{1}{ }^{2}+V S \\
& =\left(1+8 l^{3}\right)\left\{\begin{array}{c}
\left(x^{7}+6 l x^{5} y z+12 l^{2} x^{3} y z-x y^{3} z^{3}\right) X^{2} \\
\vdots \\
+\left(2 l x^{7}+12 l^{2} x^{5} y z-3 x^{2} y^{2} z^{2}-2 l x y^{3} z^{3}\right) Y Z \\
\vdots
\end{array}\right.
\end{aligned}
$$

and the equation

$$
-x y z H_{1}^{2}+V S+\left(1+8 l^{3}\right)\left(Q W-3 x^{2} y^{2} z^{2} \mathfrak{S}_{1}\right)=0
$$

is thus verified.

AdDition.-The foregoing memoir was communicated to $\mathrm{Mr}$ Salmon, and I am indebted to him for two notes, containing the extension to a curve of any order, of the preceding investigation for the case of a cubic; I reproduce this extension in the following section. 


\section{Extension of the last preceding method to a curve of any order.}

54. Consider the curve of the $m$-th order $\Upsilon=0$, and in the place of the coordinates write $\lambda x+\mu X, \lambda y+\mu Y, \lambda z+\mu Z$, where, as before, $(x, y, z)$ are the coordinates of the point of the curve, and $X, Y, Z$ current coordinates; the term involving $\lambda^{m}$ vanishes, and dividing out the factor $\mu$ the equation becomes

$$
\lambda^{m-1} D U+\frac{1}{2} \lambda^{m-2} \mu D^{2} U+\frac{1}{6} \lambda^{m-3} \mu^{2} D^{3} U+\frac{1}{24} \lambda^{m-4} \mu^{3} D^{4} U+\& c .=0 .
$$

Making the like substitution in $D^{2} U-\Pi D U=0$, the assumed equation of the fivepointic conic, the factor $\mu$ divides out and the equation becomes

$$
2 \lambda(m-1) D U+\mu D^{2} U-(\lambda P+\mu \Pi) D^{2} U=0,
$$

or, what is the same thing,

$$
\lambda D U(2(m-1)-P)+\mu\left(D^{2} U-\Pi D U\right)=0 ;
$$

and if from the two equations we eliminate $\lambda, \mu$, the result, throwing out the factor $D U$, is

$$
\left(D^{2} U-\Pi D U\right)^{m-1}-\frac{1}{2}(2(m-1)-P) D^{2} U\left(D^{2} U-\Pi D U\right)^{m-2}+\& c .=0,
$$

where all the terms after the second contain the factor $D U$; the condition in order that the equation may divide by $D U$, is consequently $2(m-1)-P=2$, or $P=2(m-2)$, the condition of a three-pointic contact. Substituting this value, and dividing by $D U$, the equation becomes

$$
-\Pi\left(D^{2} U-\Pi D U\right)^{m-2}+\frac{2}{3} D^{3} U\left(D^{2} U-\Pi D U\right)^{m-3}-\frac{1}{3} D^{4} U . D U\left(D^{2} U-\Pi D U\right)^{m-4}+\& c .=0,
$$

which will be divisible by $D U$ if $-\Pi D^{2} U+\frac{2}{3} D^{3} U$ is divisible by $D U$, and the condition for this is found to be, as before,

$$
\Pi=\frac{2}{3} \frac{1}{H} D H+\Lambda D U,
$$

where $\Lambda$ is arbitrary; we have thus the conditions of a four-pointic contact.

55. Substituting this value of $\Pi$, we see that $D^{3} U-\frac{1}{H} D H . D^{2} U$ divides by $D U$, viz. there exists an identical equation,

$$
D^{3} U-\frac{1}{H} D H . D^{2} U=I U+J . D U
$$

and hence if $U=0$,

$$
\frac{1}{D U}\left(D^{3} U-\frac{1}{H} D H \cdot D^{2} U\right)=J,
$$

where $J$ is a quadric function of $(X, Y, Z)$. I do not know the general form of this function, but $\mathrm{Mr}$ Salmon has obtained a result which may be generalized as 
follows, viz. writing for $X, Y, Z$ the values $B \nu-C \mu, C \lambda-A \nu, A \mu-B \lambda$ (where, as before, $A, B, C$ are the first derived functions of $U$ and $\lambda, \mu, \nu$ are arbitrary), the expression for $J$ is

$$
J=\frac{1}{D U}\left(D^{3} U-\frac{1}{H} D H \cdot D^{2} U\right)=\frac{3(m-2)}{(m-1)} \Phi-\frac{1}{(m-1) H} \square 9 ;
$$

a formula which will be presently useful.

56. The foregoing equation may be written

$$
\begin{aligned}
& \left(D^{2} U\right)^{m-3}\left(-\Pi D^{2} U+\frac{2}{3} D^{3} U\right) \\
+ & \left(D^{2} U\right)^{m-4} D U\left\{(m-2) \Pi^{2} D^{2} U-\frac{2}{3}(m-3) \Pi D^{3} U-\frac{1}{3} D^{4} U\right\}+\& c .(D U)^{2} \ldots=0 ;
\end{aligned}
$$

and the term $-\Pi D^{2} U+\frac{2}{3} D^{3} U$ is equal to

$$
\frac{2}{3}\left(D^{3} U-\frac{1}{H} D H \cdot D^{2} U\right)-\Lambda D U \cdot D^{2} U,=\frac{2}{3} J D U-\Lambda D U \cdot D^{2} U .
$$

Substituting this value the equation divides by $D U$, and throwing out this factor it becomes

$$
\begin{aligned}
& \left(D^{2} U\right)^{m-3}\left(\frac{2}{3} J-\Lambda D^{2} U\right) \\
+ & \left(D^{2} U\right)^{m-4}\left\{(m-2) \Pi^{2} D^{2} U-\frac{2}{3}(m-3) \Pi D^{3} U-\frac{1}{3} D^{4} U\right\}+\& \text { c. } D U=0 ;
\end{aligned}
$$

or observing that $\frac{2}{3} \Pi D^{3} U=\Pi^{2} D^{2} U+$ term containing $D U$, this may be written

$$
\left(D^{2} U\right)^{m-3}\left(\frac{2}{3} J-\Lambda D^{2} U\right)+\left(D^{2} U\right)^{m-4}\left(\Pi^{2} D^{2} U-\frac{1}{3} D^{4} U\right)+\& c . \quad D U=0 .
$$

57. If the equation divides by $D U$ we shall have a five-pointic contact; the condition for this is that

$$
-\Lambda\left(D^{2} U\right)^{2}+\frac{2}{3} J D^{2} U+\Pi^{2} D^{2} U-\frac{1}{3} D^{4} U
$$

may divide by $D U$, or more simply that

$$
-\Lambda\left(D^{2} U\right)^{2}+\frac{2}{3} J D^{2} U+\frac{4}{9}\left(\frac{1}{H} D H\right)^{2} D^{2} U-\frac{1}{3} D^{4} U
$$

may divide by $D U$, or, what is the same thing, the function in question must vanish in virtue of the substitution of the values $B \nu-C \mu, C \lambda-A \nu, A \mu-B \lambda$ in the place of $X, Y, Z$. The expression for $J$ has just been given; we have besides

$$
\left(\frac{1}{H} D H\right)^{2}=\frac{(m-1)^{4}}{H^{2}} Q_{3}^{2}, D^{4} U=-Q_{4} 9^{2},
$$

where the values of $Q_{3}{ }^{2}, Q_{4}$ are given (ante, No. 27); we have thus

58. $\frac{1}{D U}\left(D^{3} U-\frac{1}{H} D H . D^{2} U\right)=\frac{3(m-2)}{m-1} \Phi-\frac{1}{(m-1) H} \square 9$,

$$
\begin{array}{ll}
\frac{4}{9}\left(\frac{1}{H} D H\right)^{2} & =-\frac{4(m-2)^{2}}{(m-1)^{2}} \Phi+\frac{4}{3} \frac{(m-2)^{2}}{(m-1)^{2}} \frac{\square}{H} 9-\frac{4}{9} \frac{1}{(m-1)^{2}} \frac{1}{H^{2}} \Psi 9^{2}, \\
-\frac{1}{3} D^{4} U & =9^{2}\left\{-\frac{2(m-2)(m-3)}{(m-1)^{4}} \Phi H+\frac{4}{3} \frac{(m-3)}{(m-1)^{4}} \square 9-\frac{1}{3} \frac{1}{(m-1)^{4}} \Omega 9^{2}\right\},
\end{array}
$$


and

$$
D^{2} U=-\frac{1}{(m-1)^{2}} H 9^{2}
$$

whence

$$
\begin{aligned}
& \Lambda \frac{1}{(m-1)^{4}} H^{29} 9^{4}= \\
& -\frac{2}{3} \frac{1}{(m-1)^{2}} H \Im^{2}\left\{\frac{3(m-2)}{m-1} \Phi-\frac{1}{(m-1) H} \square 9\right. \\
& -\frac{1}{(m-1)^{2}} H \Im^{2}\left\{\frac{-4(m-2)^{2}}{(m-1)^{2}} \Phi+\frac{4}{3} \frac{(m-2)}{(m-1)^{2} H} \square 9-\frac{4}{9} \frac{1}{(m-1)^{2}} \frac{1}{H^{2}} \Psi 9^{2}\right\} \\
& +9^{2}\left\{-\frac{2(m-2)(m-3)}{(m-1)^{4}} \Phi H+\frac{2}{3} \frac{(m-3)}{(m-1)^{4}} \square \mathscr{S}-\frac{1}{3} \frac{1}{(m-1)^{4}} \Omega 9^{2}\right\}, \\
& =\frac{1}{9(m-1)^{4} H}(4 \Psi-3 \Omega H) \text {, }
\end{aligned}
$$

and consequently

$$
\Lambda=\frac{1}{9 H^{3}}(4 \Psi-3 \Omega H),
$$

which agrees with the result before obtained, and thus the present method gives the complete solution of the problem. 